\title{
Factors Affecting Social Exclusion, Friendship Quality, Social Competence and Emotion Management Skills and the Effect of Problem Behaviors on Related Characteristics in Adolescents
}

\author{
Meral Sert Ağır \\ Correspondence: Educational Science Department, Ataturk Education Faculty, Marmara University, Goztepe, Istanbul, \\ Turkey.
}

Received: September 6, 2019

Accepted: October 15, 2019 Online Published: October 16, 2019

doi:10.11114/jets.v7i10S.4508

URL: https://doi.org/10.11114/jets.v7i10S.4508

\begin{abstract}
This study was conducted to determine the predictive effect of the features (scale scores) related to the factors that affect social exclusion, friendship quality, social competence and emotional management skills in adolescents on adolescent problem behaviors such as smoking, alcohol use, unhappiness, hopelessness and self-harm behaviors. This research was carried out on 422 students studying at $9^{\text {th }}, 10^{\text {th }}$ and $11^{\text {th }}$ grades in 11 high schools randomly selected among the state Anatolian high schools of Kadıköy with the permission of Istanbul Governorship MNE No: 59090411-20-E.4519169 dated 21.04.2016. Data were collected through Social Exclusion, Friendship Quality, Social Competence and Emotional Management Scales and personal information form prepared by the researcher. The data were analyzed with SPSS 23 statistical software, two-way ANOVA (univariate) and logistic regression techniques. The findings showed that on the features related to social exclusion, social competence, friendship quality and emotional management skills, the following were effective: having smoker friends, having friends with negative behaviors towards others, dissatisfaction with physical appearance, perception of self-efficacy, getting along with friends, being sensitive towards daily events, having smoker family members and alcohol use the family, experiencing less economic problems in the family and participating in activities such as cinema with the family. The quality of friendship intimacy had an increasing effect on smoking and alcohol use, while the security dimension had a reducing effect on alcohol use, feeling unhappy, feeling hopeless and self-harm (bodily damage). The social exclusion, emotional management and coping with the problem dimensions had a diminishing effect on alcohol use, while negative emotions and the ability to control negative bodily reactions had a diminishing effect on self-harm behaviors. The findings suggest that, especially emotional management skills, friendship quality and social exclusion are dynamics that can determine the psycho-social risk susceptibility of adolescents. The results of the research reveal the importance of getting adolescents to gain the skills to manage friendship selection and friendship relations through studies aimed at supporting the emotional development of adolescents.
\end{abstract}

Keywords: social exclusion, friendship quality, social competence, emotion management skills, problem behaviors

\section{Introduction}

A human being is a social entity that defines and maintains his/her existence through others. The journey of life that begins in the mother's womb can be sustained by the presence of others whose roles and duties change during different periods of this journey. The differentiating needs in each period of development require a change of positions, roles and duties of others, who are parents in the first years of life, teachers in childhood, peers in adolescence (Brown, 2004) and emotional partners (spouse) and colleagues in young adulthood. Thus, each period of development allows the human to realize himself/herself through others and to continue to exist as an "individual" among others (Zimmer- Gembeck, Collins, 2006).

In the process from infancy to old age, adolescence serves as a bridge that enables the transition from dependence to independence. Before puberty, many vital actions, from what their needs should be to how they should be met, take place through the choices of the parents. Adolescence is the preparation stage for the period where decisions and outcomes depend on the individual, from the period of young adulthood until the end of life. One of the characteristic features of adolescence is the change in the positions of social environmental agents at the center of life. With adolescence, the role definitions of the family and the peers are differentiated, and peers are placed at the center of 
adolescent life as a social environment, where acquired competencies within the family are tried and reformulated (Schunk,Meece, 2005; Brody, 2004; Rose, Rudolph, 2006). Beyond physiological differentiation, the term refers to knowing and discovering oneself and others through a different window and to proving their existence to the authority figures, the adults (Bukowski, Newcomb, Hartup, 1996).

Another developmental dynamic in this period is the sudden changes in the adolescent's emotions, where they have the most difficulty in their relationships with adolescents and feel helpless about how they can react (Malatesta, 1990; Galambos, Costigan, 2003, Nurmi, 2004). The behaviors that adolescents prefer to express themselves in the family and peer environment are closely related to their emotional states (Silvers, McRae, Gabrieli, Gross, Remy, Ochsner, 2012). The ability to control emotional changes, which is also an effective characteristic in the interpersonal relationships of adults, helps adolescents manage both their peer and adult relationships (Thompson, 1991; Carlo, Crockett, Wolff, Beal, 2012). While many complex developmental differentiations, such as neurophysiological (Konrad, Firk, Uhlhaas, 2013), mental, social differentiation, etc., are the source of the change in mood states (Yurgelun, 2007), the behaviors produced by the adolescent to cope with the changes in their emotional states provide information about the competences of them (Connolly, 1989; Dusek, McIntyre, 2006) and of their social environment.

The adolescent meets the need to be valued and approved by expressing his/her thoughts and feelings about every day events or social events related to his/her own life and strengthens the definition of self (Leahy, 2001; Good, Grand, Newby-Clark, Adams, 2008).The adolescent's anxiety about whether their ideas are accepted or their feelings are understood, which accompany the effort to prove themselves in both the adult and peer worlds, may play a role in triggering the sudden and rapid change in their emotions (Susman, Rogal, Alan, 2004). Having the ability to control the change in his/her emotional processes can lead to positive development of relationships, and the other way round, it can cause problems in many areas, from family relationships to peer relationships (Anderson, Betz, 2001; Brown, Klute, 2006; McLaughlin, Megan, Garrard and Leah Somerville, 2015) and academic life (Pajares,1996; Pajares, Schunk, 2001). Considering the emotional social relationships that adolescents have by creating a special space with their peers like adults, the emotional gains of these relationships, such as being valued, accepted and cared, play a supporting role in their healthy development (Lerner, Steinberg, 2004). Therefore, while adolescents' ability to control their emotions can positively affect the quality of their friendship and t social competence (Zimmerman, Cleary, 2005), it can cause their exclusion from peer groups, being left alone and disregarded in the exact opposite situation and may affect their emotional and social development. When we discuss the issue through the impact of peers on adolescents (Leary,1990; Meuwese, Cillessen, Güroğlu, 2017) we are faced with whether peers show healthy emotional social development or the dynamics that can be produced by the way they maintain friendship relationships (Flynn, Felmlee, Conger, 2017). In other words, peers, as the leading roles in adolescent life, teach each other which behavior is the key to being accepted. The literature on adolescent behavior and adaptation problems draws attention to the peer effect on adolescents' health-threatening behaviors and states that adolescents tend to develop behaviors that may have short- or long-term adverse effects to be found valuable, adequate and special by their peers (Klein, Cornell, Konold, 2012; Corsano, Musetti, Caricati, Magnani, 2017; Veenstra, Dijkstra, Kreager, 2018). As with cigarette, alcohol, substance use or early sexual experience, which peer groups find meaningful or perceive as criteria of maturity (Harris, Duncan, Boisjoly, 2002; Kuther, 2002), it can threaten the health of adolescents during this development period and change their quality of life in later periods. However, because it can prevent the adolescent from being excluded, ignored or left alone by his/her friends, it enables him/her to achieve instant gratifying results (Rusby, Forrester, Biglan, Metzler, 2005; Gioia, 2017). In addition, when problems occur with peers or adult authority figures, such behavior may be preferred to avoid the problem, to relax or to prove himself/herself to both groups.

These behaviors that make adolescents feel worthy and valuable through short, instant and satisfactory gains, which they can consciously prefer having been influenced by whether the developmental dynamics or social environment as a consequence of the thought and feeling that they have generated solutions to their problems, even if they are unhealthy, are defined as risk-taking behaviors in the adolescence literature (Guerra, Bradshaw, 2008). In fact, adolescents may maintain these behaviors even if they have knowledge about the long-term unhealthy consequences. In other words, adolescents' decisions with respect to what pose a risk for themselves are the predictors of the behaviors that the literature identifies as risky (Gullone, Moore 2000). Adolescents may not perceive such behaviors as health-threatening risks or may consider these as affordable risks depending on the result. If these are socially accepted as adult role behaviors, their tendency towards these behaviors may increase to gain status within their peer groups by manifesting their competences (Leather, 2009). In addition, adolescents' belief in their omnipotence, that is, the presumption that nothing will happen to them or that they have control over the consequences of their behaviors, also catalyzes their inclination towards these behaviors and participation in the groups that exhibit such behaviors.

On the other hand, the behaviors, which inhibit, undermine or threaten the healthy development process yet are not perceived as health-threatening risks by adolescents, are defined as problem behaviors in the problem behavior theory 
(Jessor, 1984). According to the theory, problem behaviors can be viewed as self-directed or outward-directed, or both can be observed as nested together. The self-directed problem behaviors of adolescents may manifest themselves as such moods as depression, desperation or unhappiness, whereas the outward-directed behaviors can be observed at various dimensions ranging from smoking, alcohol use or drugs to theft and violence that can be considered within the scope of youth delinquency in legal terms (Jessor, 1987; Wilson, Jonah, 1988, Jessor, 2001). The problem behavior theory emphasizes that the problematic behaviors of adolescents emerge by the interaction of three systems related to them, their environment and their behaviors (Siyez, Aysan, 2007). According to the theory, adolescents' tendency towards health-threatening behaviors to cope with developmental adaptation problems is shown by their psycho-social risk-taking tendencies, while this tendency is determined by the balance between protective and triggering factors (Jessor, 2014). While the sufficiency of the social environment to support healthy development stands out as a protective factor (Siyez, 2008), its development-inhibiting or process-violating impact, when it takes on a triggering function, increases the tendency towards problematic behaviors (Duncan, Duncan, Strycker, 2000; Shope, Waller, Raghunathan, Patil, 2001).

In conclusion, it is seen that adolescent behaviors can affect their own characteristics, family and peer groups (Brody,1998), their problems and their coping skills as their social environment and function of their behaviors. Given the effect of the sudden emotion changes and the peer groups on the behavioral preferences of adolescents, psycho-social risk-taking tendency emerges depending on whether these two factors have protective or triggering functions (Guerra, \& Bradshaw, 2008; Wilkinson, Marmot, 2003). In the light of the related literature, this study was conducted to examine adolescents' emotion management skills (Yeager, 2017), social competency perceptions and social isolation (Lodder, Scholte, Goossens, Verhagen, 2017) and peer quality characteristics (Mason, Zaharakis, Rusby, Westling, Light, Mennis, Flay, 2017), which identify their problems with their social environments, in terms of problematic behaviors to contribute to the studies aimed at improving the quality of life of future adults by ensuring the healthy development of adolescents. In this study, taking into consideration that the two dynamics of adolescent behaviors and the environment can both be the cause and effect of each other, the factors affecting the respective characteristics and the impact of the respective characteristics on problem behaviors were explored. In this context, the main purpose of this study was to examine the factors affecting social isolation, peer quality, need for belonging, social competency and emotion management skills of adolescents and the predictive impacts of these characteristics on smoking, alcohol use, self-harming (bodily damage), unhappiness and depression, which are among problem behaviors.

\subsection{Purpose of the Study}

The aim of this study was to investigate the factors that affect social exclusion, friendship quality, social competence and emotion management skills in adolescents and the effect of the related characteristics (scale scores) on adolescents' problem behaviors. Accordingly, the research sought answers to the following questions:

1. What are the factors affecting social exclusion in adolescents?

2. What are the factors affecting adolescents' quality of friendship?

3. What are the factors affecting the social competence of adolescents?

4. What are the factors affecting adolescents' emotion management skills?

5. To what extent can adolescents' relevant characteristics (scale scores) predict their smoking, alcohol use, self-harm (bodily damage), unhappiness, negative mood and absenteeism?

\subsection{Limitations of the Research}

The research results were limited to the young people who formed the study group. Furthermore, the research was limited to the relationship between smoking, alcohol use, self-harm (bodily damage), feeling unhappy and bad among the problematic behaviors of the adolescents and social exclusion, friendship quality, social competence and emotion management skills. The data were analyzed in line with the main aim of the research; for example, the question regarding whether there was a difference in terms of demographic characteristics such as gender or age was excluded from the scope of the research.

\section{Method}

This section contains information about the research model, study group, data collection tools and techniques used in data analysis.

\subsection{Research Model}

This research was based on the relational screening model. 


\subsection{Study Group}

This research was carried out in 11 high schools randomly selected among the public Anatolian and vocational high schools of Kadıköy with the permission of Istanbul Governorship MNE No: 59090411-20-E.4519169 dated 21.04.2016. To conduct the research in a healthy way, appointments were made with the administrations of the relevant schools before the research, and the $12^{\text {th }}$ grades of the high schools were excluded as they were preparing for the entrance exam to higher education. Of the 467 students randomly selected from among the $9^{\text {th }}, 10^{\text {th }}$ and $11^{\text {th }}$ grades of the high schools, the data of a total of 422 students who participated in the research (196 males and 226 females) were analyzed. The research was conducted under the supervision of the researcher and 4 university senior students, and the participating students were informed regarding the aim of the research to ensure their voluntary participation. Data on the demographic characteristics of the students are presented in Table 1.

Table 1. Demographic Characteristics of the Students

\begin{tabular}{|c|c|c|c|}
\hline $\mathrm{n}=431$ & & $\mathrm{n}$ & $\%$ \\
\hline \multirow{2}{*}{ Gender } & Male & 196 & 46.4 \\
\hline & Female & 226 & 53.6 \\
\hline \multirow{7}{*}{ Age } & 11 & 1 & .2 \\
\hline & 13 & 1 & .2 \\
\hline & 14 & 27 & 6.4 \\
\hline & 15 & 182 & 42.9 \\
\hline & 16 & 97 & 22.9 \\
\hline & 17 & 87 & 20.5 \\
\hline & 18 & 29 & 6.8 \\
\hline \multirow{5}{*}{ Grade } & Preparatory class & 3 & .7 \\
\hline & 9 & 238 & 56.3 \\
\hline & 10 & 69 & 16.3 \\
\hline & 11 & 87 & 20.6 \\
\hline & 12 & 26 & 6.1 \\
\hline \multirow{5}{*}{ School achievement } & Very good & 32 & 7.7 \\
\hline & Good & 192 & 46.2 \\
\hline & Not bad & 168 & 40.4 \\
\hline & $\mathrm{Bad}$ & 17 & 4.1 \\
\hline & Very bad & 7 & 1.7 \\
\hline \multirow{5}{*}{ Mother's educational status } & Illiterate & 7 & 1.7 \\
\hline & Primary school graduate & 98 & 23.3 \\
\hline & Secondary school graduate & 76 & 18.1 \\
\hline & High school graduate & 120 & 28.5 \\
\hline & University graduate & 120 & 28.5 \\
\hline \multirow{5}{*}{ Father's educational status } & Illiterate & 3 & .7 \\
\hline & Primary school graduate & 64 & 15.2 \\
\hline & Secondary school graduate & 77 & 18.2 \\
\hline & High school graduate & 141 & 33.4 \\
\hline & University graduate & 137 & 32.5 \\
\hline
\end{tabular}




\subsection{Data Collection Tools}

Data were obtained by using the "Emotion Management Skills Scale", "Perceived Social Competence Scale", "Friendship Quality Scale", "Social Exclusion Scale", "Belonging Scale" and "Personal Information Form".

\subsubsection{Emotion Management Skills Scale}

The scale developed by Rezzan Çeçen is a 5-point Likert-type self-assessment scale consisting of a total of 28 items. The scale consists of five sub-scales: "Expressing Emotions Verbally", "Recognizing and Accepting Emotions", "Showing Emotions as They Are", "Controlling Negative Physical Reactions", "Coping" and "Managing Anger". High scores indicate being competent in one's ability to manage his/her emotions. The internal consistency coefficient (cronbach's alpha) for EMSS was found as 0.83(Çeçen, 2006).

\subsubsection{Perceived Social Competence Scale}

The scale developed by Anderson-Butcher et al. to measure students' perception of social competence was adapted to Turkish by Sarıçam et al. Consisting of one dimension and 6 questions, the scale is arranged in the form of a 5-point Likert scale. The internal consistency reliability coefficient of the scale was found as 0.80 , while in a study with similar group it was found as 0.78(Sarıçam, Akın, Akın, Çardak, 2013).

\subsubsection{Friendship Quality Scale}

The scale developed by Thien et al. to evaluate the effects of friendship on individuals was adapted to Turkish by Akın et al. The scale is a 6-point Likert scale consisting of 21 items and four sub-dimensions - safety, intimateness, acceptance and assistance (Akın, Karduz Adam, Akın, 2014).

\subsubsection{Social Exclusion Scale}

The five-point Likert-type scale consists of 11 questions. There are two sub-dimensions of the scale: being ignored and exclusion. The scale gives scores based on the sub-dimensions and yields total scores. Cronbach's alpha internal consistency reliability coefficient was found as 0.93 for the being ignored sub-dimension, 0.90 for the exclusion sub-dimension and 0.89 for the whole scale. Corrected item-test correlations of the scale ranged between 0.51 and 0.70 (Akın, Doğan, Gönülalan, Atik, Çebiş, Akın, 2014).

\subsubsection{Personal Information Form}

The Personal Information Form was prepared by the researcher within the scope of the literature related to problematic behaviors in adolescents.

As for the questions about smoking and alcohol use of adolescents, smoking and alcohol expressions were not used; related questions are arranged as follows.

unhealthy/unsuitable substance for sale under 18 years of age (available for adults at the market)

unhealthy/unsuitable drink for sale under 18 years of age (not available for adults at the market after a certain hour)

\subsection{Data Analysis}

The data were analyzed with SPSS 23 software, and the level of confidence was at 95\%. Two-way ANOVA (Univariate) and Logistic Regression tests were used in the study. Two-way ANOVA is an analysis method that considers the correlation of independent variables with each other while examining the effect of independent variables on a dependent variable (Kalayc1 2006). Logistic regression analysis has recently become a widespread method, especially in the social sciences. In most socio-economic studies conducted to reveal cause-effect relationships, some variables consist of two-level data such as positive-negative, successful-unsuccessful, yes-no and satisfied-dissatisfied. In case such a dependent variable consists of two-level or multi-level categorical data, Logistic Regression Analysis has an important place in the study of cause-effect relationship between the dependent variable and the independent variable(s) (Agresti, 1996). In logistic regression analysis, which aims to classify and investigate the relationships between dependent and independent variables, the dependent variable generates categorical data and takes discrete values. There is no requirement for all or some of the independent variables to be continuous or categorical variables. Logistic regression analysis is an alternative method to discriminant analysis and cross-tables when regression analysis does not provide some assumptions such as normality and presence of a common covariance. In addition to its applicability even when the dependent variable is a discrete variable with two levels such as 0 and 1 or more, its mathematical flexibility and ease of interpretability increase the interest in this method (Hosmer Jr, Lemeshow, May, 2008). The effects of explanatory variables on the dependent variable are obtained as probabilities to determine the probabilities of risk factors (Archer, Lemeshow, Hosmer, 2007). 


\section{Findings}

In this section, the findings on the descriptive characteristics of the study group and the results of the analyses are stated.

\subsection{Descriptive Findings}

This section includes the findings related to the frequency of problem behaviors of the students involved in the study.

Table 2. Personal Information about Adolescent's life

\begin{tabular}{|c|c|c|c|}
\hline $\mathrm{n}=431$ & & $\mathrm{n}$ & $\%$ \\
\hline \multirow[t]{4}{*}{ Getting along with peers } & Very good & 118 & 27.6 \\
\hline & Pretty good & 254 & 59.5 \\
\hline & Not very good & 51 & 11.9 \\
\hline & Always bad & 4 & .9 \\
\hline \multirow[t]{4}{*}{ Interest/curiosity in/about daily problems } & Quite a lot & 189 & 44.1 \\
\hline & A little & 188 & 43.8 \\
\hline & Not much & 47 & 11.0 \\
\hline & Never & 5 & 1.2 \\
\hline \multirow[t]{4}{*}{ Being competent in decisions about his/her own life } & Very good & 71 & 16.6 \\
\hline & Pretty good & 262 & 61.2 \\
\hline & Not very good & 85 & 19.9 \\
\hline & Always bad & 10 & 2.3 \\
\hline \multirow[t]{4}{*}{ Opinion about his/her body (physical appearance) } & Very satisfied & 79 & 18.5 \\
\hline & Pretty satisfied & 204 & 47.8 \\
\hline & Not very satisfied & 120 & 28.1 \\
\hline & Very dissatisfied & 24 & 5.6 \\
\hline \multirow[t]{4}{*}{ Opinion about his/her efficiencies/competencies } & I am very talented & 123 & 28.9 \\
\hline & I am quite talented & 214 & 50.4 \\
\hline & I am not very talented & 77 & 18.1 \\
\hline & I am not talented & 11 & 2.6 \\
\hline
\end{tabular}

Table 3. Family, Environmental Information

\begin{tabular}{|c|c|c|c|}
\hline$n=431$ & & $\mathrm{n}$ & $\%$ \\
\hline \multirow{5}{*}{$\begin{array}{l}\text { Can you easily go to cinema or theater or participate in any social } \\
\text { activity with your family? }\end{array}$} & Always & 181 & 42.4 \\
\hline & Frequently & 64 & 15.0 \\
\hline & Sometimes & 73 & 17.1 \\
\hline & Rarely & 70 & 16.4 \\
\hline & Never & 39 & 9.1 \\
\hline \multirow{5}{*}{$\begin{array}{l}\text { Do you think that you and your family live without economic } \\
\text { problems? }\end{array}$} & Always & 198 & 46.4 \\
\hline & Frequently & 132 & 30.9 \\
\hline & Sometimes & 57 & 13.3 \\
\hline & Rarely & 20 & 4.7 \\
\hline & Never & 20 & 4.7 \\
\hline
\end{tabular}


Table 4. Harmful Habit Information

\begin{tabular}{|c|c|c|c|}
\hline$n=431$ & & $\mathrm{n}$ & $\%$ \\
\hline \multirow{6}{*}{ Harmful habit status } & Obesity & 14 & 3.2 \\
\hline & Smoking & 98 & 22.7 \\
\hline & Alcohol & 98 & 22.7 \\
\hline & Obesity in the family & 18 & 4.2 \\
\hline & Smoking in the family & 170 & 39.4 \\
\hline & Alcohol in the family & 91 & 21.1 \\
\hline \multirow{6}{*}{ Damage caused by harmful habit } & Obesity & 6 & 1.4 \\
\hline & Smoking & 22 & 5.1 \\
\hline & Alcohol & 19 & 4.4 \\
\hline & Obesity in the family & 4 & .9 \\
\hline & Smoking in the family & 38 & 8.8 \\
\hline & Alcohol in the family & 18 & 4.2 \\
\hline
\end{tabular}

Table 5. Harmful Habit and Behavior Information

\begin{tabular}{|c|c|c|c|}
\hline $\mathrm{n}=431$ & & $\mathrm{n}$ & $\%$ \\
\hline \multirow{4}{*}{ Smoking } & No, never & 247 & 60.0 \\
\hline & Yes, 1-2 times & 75 & 18.2 \\
\hline & Several times & 26 & 6.3 \\
\hline & More than a few times & 64 & 15.5 \\
\hline \multirow{4}{*}{ Have you ever felt bad? } & Many times & 109 & 25.6 \\
\hline & Quite a lot & 106 & 24.9 \\
\hline & A little & 164 & 38.6 \\
\hline & Hardly ever & 46 & 10.8 \\
\hline \multirow{4}{*}{ Have you ever felt unhappy? } & Many times & 88 & 20.7 \\
\hline & Quite a lot & 84 & 19.8 \\
\hline & A little & 155 & 36.5 \\
\hline & Hardly ever & 98 & 23.1 \\
\hline \multirow{2}{*}{ Did you do anything to harm your body? } & Yes & 72 & 17.3 \\
\hline & No & 344 & 82.7 \\
\hline \multirow{3}{*}{ Alcohol } & Yes & 117 & 33.3 \\
\hline & No & 234 & 66.7 \\
\hline & Myself & 11 & 8.9 \\
\hline \multirow{4}{*}{ Friends smoking } & None & 69 & 16.4 \\
\hline & Several & 91 & 21.6 \\
\hline & Some & 138 & 32.8 \\
\hline & Majority & 123 & 29.2 \\
\hline \multirow{4}{*}{ Friends treating others badly } & None & 148 & 35.2 \\
\hline & Several & 140 & 33.3 \\
\hline & Some & 98 & 23.3 \\
\hline & Majority & 35 & 8.3 \\
\hline
\end{tabular}


Table 6. Descriptive Statistics of Scale Scores

\begin{tabular}{lccccc}
\hline $\mathrm{n}=431$ & Minimum & Maximum & Mean & Std. & Level \% \\
\hline Social Exclusion & 11 & 54 & 22.02 & 8.38 & 40.78 \\
\hline Being ignored & 5 & 25 & 7.37 & 3.96 & 29.49 \\
\hline Exclusion & 6 & 30 & 14.65 & 6.28 & 48.83 \\
\hline Friendship Quality & 25 & 126 & 89.34 & 20.08 & 70.90 \\
\hline Safety & 8 & 48 & 30.84 & 8.38 & 64.25 \\
\hline Intimacy & 6 & 36 & 27.05 & 6.90 & 75.15 \\
\hline Acceptance & 4 & 24 & 18.42 & 4.49 & 76.75 \\
\hline Help & 2 & 12 & 8.88 & 2.78 & 73.98 \\
\hline Social Competence & 6 & 30 & 24.81 & 4.83 & 82.71 \\
\hline Emotional Management Skills & 44 & 130 & 92.42 & 12.79 & 71.09 \\
\hline Verbal Expression Ability & 7 & 35 & 23.56 & 6.08 & 67.31 \\
\hline Showing Emotions as They Are & 6 & 30 & 20.27 & 4.46 & 67.56 \\
\hline Ability to Control Negative Physical Reactions & 4 & 20 & 12.58 & 3.44 & 62.89 \\
\hline Coping & 4 & 20 & 12.87 & 3.20 & 64.35 \\
\hline Anger Management & 3 & 14 & 8.65 & 1.96 & 61.80
\end{tabular}

3.2 Two-Way ANOVA Analysis Result About Factors Affecting Students' Social Exclusion, Friendship Quality, Social Competence, and Emotional Management Skills

Table 7. Factors Affecting Students' Social Exclusion

\begin{tabular}{|c|c|c|c|c|c|}
\hline \multirow{2}{*}{ Model } & & \multicolumn{2}{|c|}{ Social Exclusion } & \multirow{2}{*}{$\mathrm{F}$} & \multirow{2}{*}{$\mathrm{p}$} \\
\hline & & Mean & Std. Deviation & & \\
\hline \multirow{2}{*}{ Smoking in the family } & No & 22.26 & 8.35 & \multirow{2}{*}{0.716} & \multirow{2}{*}{0.398} \\
\hline & Yes & 21.66 & 8.44 & & \\
\hline \multirow{3}{*}{ Alcohol in the family } & No & 22.10 & 8.41 & \multirow{3}{*}{0.058} & \multirow{3}{*}{0.811} \\
\hline & Yes & 21.73 & 8.32 & & \\
\hline & None & 21.71 & 8.23 & & \\
\hline \multirow{3}{*}{ Friends smoking } & A few or less & 22.35 & 8.50 & \multirow{3}{*}{6.203} & \multirow{3}{*}{$0.002 *$} \\
\hline & Some & 21.54 & 8.10 & & \\
\hline & Majority & 21.75 & 8.47 & & \\
\hline \multirow{3}{*}{ Friends treating others badly } & None & 21.26 & 8.42 & \multirow{3}{*}{4.418} & \multirow{3}{*}{$0.013^{*}$} \\
\hline & Several & 22.08 & 7.66 & & \\
\hline & Several and more & 22.62 & 9.17 & & \\
\hline \multirow{3}{*}{ Getting along with peers } & Very good & 18.83 & 6.91 & \multirow{3}{*}{8.912} & \multirow{3}{*}{$0.000^{*}$} \\
\hline & Pretty good & 21.94 & 7.75 & & \\
\hline & Not very good & 28.89 & 9.95 & & \\
\hline \multirow{5}{*}{ Interest/curiosity in/about daily problems } & Quite a lot & 20.79 & 7.87 & \multirow{5}{*}{3.451} & \multirow{5}{*}{$0.033^{*}$} \\
\hline & A little & 23.11 & 8.50 & & \\
\hline & Not much & 22.90 & 9.25 & & \\
\hline & I am quite talented & 21.05 & 7.85 & & \\
\hline & I am not very talented & 24.24 & 9.09 & & \\
\hline \multirow{3}{*}{ Opinion about his/her body } & Very satisfied & 18.44 & 7.50 & \multirow{3}{*}{4.308} & \multirow{3}{*}{$0.015^{*}$} \\
\hline & Pretty satisfied & 21.29 & 7.45 & & \\
\hline & Not very satisfied & 25.11 & 9.13 & & \\
\hline
\end{tabular}

Model; $\mathrm{F}=2.498, \mathrm{p}<0.05, \mathrm{R}^{2}=0.311$

$* \mathrm{p}<0.05$ significant effect, $\mathrm{p}>0.05$ no significant effect 
The model established to determine the factors affecting the Social Exclusion of Students was statistically significant $(\mathrm{F}=2.498$, $\mathrm{p}<0.05)$ ( Exclusion perception levels of those with a few or fewer smoker friends are highest (22.35). The negative behaviors of students' friends towards others affect Social Exclusion $(\mathrm{F}=8.912, \mathrm{p}<0.05)$. Those with friends who treat others badly have the highest level of Social Exclusion perception (22.62). Students' level of getting along with peers affects Social Exclusion $(\mathrm{F}=4.418, \mathrm{p}<0.05)$. Social Exclusion perception level is the highest among those who cannot get along well with their peers (28.89). The level of common sense of students' daily problems affects Social Exclusion ( $\mathrm{F}=3.451$, $\mathrm{p}<0.05)$. The Social Exclusion perception levels of those who approach daily problems with some common sense are the highest (23.11). The level of satisfaction that students feel about their opinion affects Social Exclusion ( $\mathrm{F}=3.451$, $\mathrm{p}<0.05)$. The level of Social Exclusion perception, where the opinion is not very satisfactory, is the highest (25.11).

Table 8. Factors Affecting Students' Being Ignored

\begin{tabular}{|c|c|c|c|c|c|}
\hline \multirow{2}{*}{ Model } & & \multicolumn{2}{|c|}{ Being ignored } & \multirow[b]{2}{*}{$\mathrm{F}$} & \multirow[b]{2}{*}{$\mathrm{p}$} \\
\hline & & Mean & Std.Deviation & & \\
\hline \multirow{2}{*}{ Smoking in the family } & No & 7.26 & 3.73 & \multirow{2}{*}{0.020} & \multirow{2}{*}{0.888} \\
\hline & Yes & 7.55 & 4.30 & & \\
\hline \multirow{2}{*}{ Alcohol in the family } & No & 7.29 & 3.76 & \multirow{2}{*}{0.040} & \multirow{2}{*}{0.842} \\
\hline & Yes & 7.70 & 4.63 & & \\
\hline \multirow{3}{*}{ Friends smoking } & A few or less & 7.37 & 4.15 & \multirow{3}{*}{6.370} & \multirow{3}{*}{$0.002 *$} \\
\hline & Some & 7.27 & 3.69 & & \\
\hline & Majority & 7.41 & 4.04 & & \\
\hline \multirow{5}{*}{ Friends treating others badly } & None & 6.84 & 3.70 & \multirow{5}{*}{5.198} & \multirow{5}{*}{$0.006^{*}$} \\
\hline & Several & 7.31 & 4.13 & & \\
\hline & Several and more & 8.05 & 4.08 & & \\
\hline & Several & 7.05 & 3.77 & & \\
\hline & Several and more & 7.53 & 4.68 & & \\
\hline \multirow{5}{*}{ Getting along with peers } & Very good & 6.22 & 3.03 & \multirow{5}{*}{8.704} & \multirow{5}{*}{$0.000 *$} \\
\hline & Pretty good & 7.25 & 3.66 & & \\
\hline & Not very good & 10.27 & 5.40 & & \\
\hline & Frequently & 7.34 & 3.48 & & \\
\hline & Occasionally and less & 6.84 & 3.29 & & \\
\hline
\end{tabular}

Model; $\mathrm{F}=2.448, \mathrm{p}<0.05, \mathrm{R}^{2}=0.272$

* $\mathrm{p}<0.05$ significant effect, $\mathrm{p}>0.05$ no significant effect

The model established to determine the factors affecting Students' Being Ignored is statistically significant (F=2.498, $\mathrm{p}<0.05$ ) (see Table 8). Having smoker friends affects Being Ignored ( $\mathrm{F}=6.370, \mathrm{p}<0.05)$. Those with mostly smoker friends have the highest level of perception of Being Ignored (7.41). The negative behaviors of friends towards others affect Being Ignored $(\mathrm{F}=5.198, \mathrm{p}<0.05)$. Those with friends who treat others badly have the highest level of perception of Being Ignored (8.05). Students' getting along with their peers affects Being Ignored $(\mathrm{F}=8.704, \mathrm{p}<0.05)$. Those who do not get along well with their peers have the highest level of perception of Being Ignored (10.27). 
Table 9. Factors Affecting Students' Exclusion

\begin{tabular}{|c|c|c|c|c|c|}
\hline \multirow{2}{*}{ Model } & & \multicolumn{2}{|c|}{ Exclusion } & \multirow{2}{*}{$\mathrm{F}$} & \multirow{2}{*}{$\mathrm{p}$} \\
\hline & & Mean & Std.Deviation & & \\
\hline \multirow{2}{*}{ Smoking in the family } & No & 15.00 & 6.39 & \multirow{2}{*}{1.312} & \multirow{2}{*}{0.253} \\
\hline & Yes & 14.11 & 6.07 & & \\
\hline \multirow{4}{*}{ Alcohol in the family } & No & 14.82 & 6.38 & \multirow{4}{*}{0.036} & \multirow{4}{*}{0.850} \\
\hline & Yes & 14.02 & 5.88 & & \\
\hline & Several & 13.85 & 6.15 & & \\
\hline & None & 14.60 & 6.05 & & \\
\hline \multirow{3}{*}{ Friends smoking } & A few or less & 14.98 & 6.30 & \multirow{3}{*}{2.936} & \multirow{3}{*}{0.055} \\
\hline & Some & 14.27 & 6.28 & & \\
\hline & Majority & 14.33 & 6.17 & & \\
\hline \multirow{3}{*}{ Friends treating others badly } & None & 14.42 & 6.38 & \multirow{3}{*}{2.587} & \multirow{3}{*}{0.077} \\
\hline & Several & 14.77 & 5.81 & & \\
\hline & Several and more & 14.57 & 6.68 & & \\
\hline \multirow{3}{*}{ Getting along with peers } & Very good & 12.61 & 5.73 & \multirow{3}{*}{4.482} & \multirow{3}{*}{$0.012 *$} \\
\hline & Pretty good & 14.69 & 6.05 & & \\
\hline & Not very good & 18.62 & 6.63 & & \\
\hline \multirow{3}{*}{ Common sense level of daily problems } & Quite a lot & 13.73 & 6.05 & \multirow{3}{*}{4.654} & \multirow{3}{*}{$0.010^{*}$} \\
\hline & A little & 15.54 & 6.34 & & \\
\hline & Not much & 15.02 & 6.49 & & \\
\hline \multirow{8}{*}{ Feeling about opinions } & Very satisfied & 12.05 & 5.90 & \multirow{8}{*}{4.439} & \multirow{8}{*}{$0.013^{*}$} \\
\hline & Pretty satisfied & 14.59 & 5.94 & & \\
\hline & Not very satisfied & 16.20 & 6.50 & & \\
\hline & Not very good & 15.62 & 6.36 & & \\
\hline & I am quite talented & 14.23 & 5.83 & & \\
\hline & I am not very talented & 17.43 & 6.36 & & \\
\hline & Frequently & 15.92 & 6.53 & & \\
\hline & Occasionally and less & 14.16 & 5.95 & & \\
\hline
\end{tabular}

Model; $\mathrm{F}=2.044, \mathrm{p}<0.05, \mathrm{R}^{2}=0.270$

$* \mathrm{p}<0.05$ significant effect, $\mathrm{p}>0.05$ no significant effect

The model established to determine the factors affecting the Exclusion of Students is statistically significant $(\mathrm{F}=2,044$, $\mathrm{p}<0,05)$ (see Table 9). Students' getting along with their peers affects Exclusion $(\mathrm{F}=4.482, \mathrm{p}<0.05)$. Exclusion level is the highest among those who do not get along very well with their peers (7.41). The level of common sense of students' daily problems affects Exclusion $(\mathrm{F}=4.654, \mathrm{p}<0.05)$. The Exclusion perception level of those who approach daily problems with some common sense is the highest (15.54). The perception that students feel about their opinion affects Exclusion $(\mathrm{F}=4.439, \mathrm{p}<0.05)$. Those who do not find their opinion very satisfactory have the highest level of Exclusion perception (15.62). 
Table 10. Factors affecting students' Friendship Quality Scale intimacy dimension

\begin{tabular}{|c|c|c|c|c|c|}
\hline \multirow{2}{*}{ Model } & & \multicolumn{2}{|c|}{ Intimacy } & \multirow{2}{*}{$\mathrm{F}$} & \multirow{2}{*}{$\mathrm{p}$} \\
\hline & & Mean & Std.Deviation & & \\
\hline \multirow{2}{*}{ Smoking in the family } & No & 26.46 & 7.19 & \multirow{2}{*}{2.319} & \multirow{2}{*}{0.129} \\
\hline & Yes & 27.96 & 6.35 & & \\
\hline \multirow{2}{*}{ Alcohol in the family } & No & 26.85 & 7.07 & \multirow{2}{*}{0.888} & \multirow{2}{*}{0.347} \\
\hline & Yes & 27.81 & 6.23 & & \\
\hline \multirow{3}{*}{ Friends smoking } & A few or less & 26.18 & 7.51 & \multirow{3}{*}{3.327} & \multirow{3}{*}{$0.038 *$} \\
\hline & Some & 27.38 & 6.48 & & \\
\hline & Majority & 28.08 & 6.33 & & \\
\hline \multirow{3}{*}{ Friends treating others badly } & None & 27.16 & 7.03 & \multirow{3}{*}{0.923} & \multirow{3}{*}{0.399} \\
\hline & Several & 26.83 & 6.54 & & \\
\hline & Several and more & 27.33 & 7.13 & & \\
\hline \multirow{3}{*}{ Getting along with peers } & Very good & 28.98 & 6.87 & \multirow{3}{*}{3.060} & \multirow{3}{*}{$0.049 *$} \\
\hline & Pretty good & 26.92 & 6.67 & & \\
\hline & Not very good & 23.64 & 6.63 & & \\
\hline \multirow{3}{*}{ Common sense level of daily problems } & Quite a lot & 28.21 & 6.69 & \multirow{3}{*}{2.842} & \multirow{3}{*}{0.060} \\
\hline & A little & 25.99 & 6.76 & & \\
\hline & Not much & 26.35 & 7.50 & & \\
\hline
\end{tabular}

Model; $\mathrm{F}=1.597, \mathrm{p}<0.05, \mathrm{R}^{2}=0.224$

${ }^{*} \mathrm{p}<0.05$ significant effect, $\mathrm{p}>0.05$ no significant effect

The model established to determine the factors affecting the Intimacy of Students is statistically significant $(\mathrm{F}=1,597$, $\mathrm{p}<0,05)$ (see Table 10). Having smoker friends affects Intimacy $(\mathrm{F}=3.327, \mathrm{p}<0.05)$. Those with mostly smoker friends have the highest level of Intimacy perception (28.08). The level of getting along with peers of students affects Intimacy level $(\mathrm{F}=3.060, \mathrm{p}<0.05)$. Intimacy perception level is the highest among those who get along very well with their peers (28.98). The frequency of students thinking that their families have no economic distress affects Intimacy ( $\mathrm{F}=3.398$, $\mathrm{p}<0.05)$. Intimacy perception level is the highest among those who think that they experience economic distress in their family sometimes or less (27.43).

Table 11. Factors Affecting Students' Social Competence

\begin{tabular}{|c|c|c|c|c|c|}
\hline \multirow{2}{*}{ Model } & & \multicolumn{2}{|c|}{ Social Competence } & \multirow{2}{*}{$\mathrm{F}$} & \multirow{2}{*}{$\mathrm{p}$} \\
\hline & & Mean & Std.Deviation & & \\
\hline \multirow{2}{*}{ Smoking in the family } & No & 24.39 & 5.15 & \multirow{2}{*}{10.652} & \multirow{2}{*}{$0.001 *$} \\
\hline & Yes & 25.46 & 4.23 & & \\
\hline \multirow{2}{*}{ Alcohol in the family } & No & 24.82 & 4.92 & \multirow{2}{*}{3.800} & \multirow{2}{*}{0.052} \\
\hline & Yes & 24.78 & 4.53 & & \\
\hline \multirow{3}{*}{ Friends smoking } & A few or less & 24.51 & 5.06 & \multirow{3}{*}{1.914} & \multirow{3}{*}{0.150} \\
\hline & Some & 25.39 & 4.48 & & \\
\hline & Majority & 24.67 & 4.98 & & \\
\hline \multirow{3}{*}{ Friends treating others badly } & None & 24.90 & 4.68 & \multirow{3}{*}{0.861} & \multirow{3}{*}{0.424} \\
\hline & Several & 24.72 & 4.68 & & \\
\hline & Several and more & 24.90 & 5.25 & & \\
\hline \multirow{3}{*}{ Getting along with peers } & Very good & 26.23 & 5.10 & \multirow{3}{*}{10.347} & \multirow{3}{*}{$0.000^{*}$} \\
\hline & Pretty good & 25.03 & 4.32 & & \\
\hline & Not very good & 20.98 & 4.36 & & \\
\hline \multirow{3}{*}{ Common sense level of daily problems } & Quite a lot & 25.86 & 4.52 & \multirow{3}{*}{3.828} & \multirow{3}{*}{$0.023^{*}$} \\
\hline & A little & 24.21 & 4.81 & & \\
\hline & Not much & 23.00 & 5.19 & & \\
\hline \multirow{6}{*}{ Feeling about opinions } & Very satisfied & 25.68 & 5.19 & \multirow{6}{*}{0.050} & \multirow{6}{*}{0.951} \\
\hline & Pretty satisfied & 24.99 & 4.70 & & \\
\hline & Not very satisfied & 24.10 & 4.78 & & \\
\hline & Probably yes & 24.59 & 4.89 & & \\
\hline & Probably no & 24.87 & 4.95 & & \\
\hline & Occasionally and less & 25.46 & 4.43 & & \\
\hline
\end{tabular}

Model; $\mathrm{F}=2.401, \mathrm{p}<0.05, \mathrm{R}^{2}=0.300$

${ }^{*} \mathrm{p}<0.05$ significant effect, $\mathrm{p}>0.05$ no significant effect 
The model established to determine the factors affecting the Social Competence of Students is statistically significant $(\mathrm{F}=2.401, \mathrm{p}<0.05)$ (see Table 11). Having smoker family members affects Social Competence $(\mathrm{F}=10.652, \mathrm{p}<0.05)$. The highest level of Social Competence perception is found among those with smoker family members (25.46). The level of getting along with peers affects Social Competence $(F=10.347, p<0.05)$. Social Competence perception level of those who get along well with their peers is the highest (26.23). The level of common sense of students' daily problems affects Social Competence $(\mathrm{F}=10.347, \mathrm{p}<0.05)$. Social Competence perception level is the highest among those who deal with problems with common sense (25.86).

Table 12. Factors affecting students' ability to express themselves verbally among Emotional Management Skills Sub-dimensions

\begin{tabular}{|c|c|c|c|c|c|}
\hline \multirow{2}{*}{ Model } & & \multicolumn{2}{|c|}{ Verbal Expression Ability } & \multirow[t]{2}{*}{$\mathrm{F}$} & \multirow{2}{*}{$\mathrm{p}$} \\
\hline & & Mean & Std.Deviation & & \\
\hline \multirow{2}{*}{ Smoking in the family } & No & 23.30 & 5.86 & \multirow{2}{*}{0.039} & \multirow{2}{*}{0.843} \\
\hline & Yes & 23.95 & 6.41 & & \\
\hline \multirow{2}{*}{ Alcohol in the family } & No & 23.33 & 5.89 & \multirow{2}{*}{4.308} & \multirow{2}{*}{$0.039 *$} \\
\hline & Yes & 24.42 & 6.72 & & \\
\hline \multirow{3}{*}{ Friends smoking } & A few or less & 23.28 & 6.31 & \multirow{3}{*}{2.887} & \multirow{3}{*}{0.058} \\
\hline & Some & 24.62 & 5.18 & & \\
\hline & Majority & 23.00 & 6.51 & & \\
\hline \multirow{3}{*}{ Friends treating others badly } & None & 24.22 & 5.94 & \multirow{3}{*}{2.942} & \multirow{3}{*}{0.055} \\
\hline & Several & 23.33 & 5.60 & & \\
\hline & Several and more & 23.20 & 6.58 & & \\
\hline \multirow{3}{*}{ Getting along with peers } & Very good & 25.07 & 6.11 & \multirow{3}{*}{6.342} & \multirow{3}{*}{$0.002 *$} \\
\hline & Pretty good & 23.63 & 5.64 & & \\
\hline & Not very good & 20.20 & 6.91 & & \\
\hline \multirow{5}{*}{ Common sense level of daily problems } & Quite a lot & 24.28 & 6.00 & \multirow{5}{*}{0.641} & \multirow{5}{*}{0.528} \\
\hline & A little & 23.04 & 5.92 & & \\
\hline & Not much & 22.42 & 6.52 & & \\
\hline & I am quite talented & 24.97 & 6.07 & & \\
\hline & I am not very talented & 21.85 & 5.92 & & \\
\hline \multirow{3}{*}{ Feeling about opinions } & Very satisfied & 24.96 & 6.05 & \multirow{3}{*}{0.193} & \multirow{3}{*}{0.824} \\
\hline & Pretty satisfied & 24.24 & 5.60 & & \\
\hline & Not very satisfied & 21.94 & 6,45 & & \\
\hline \multirow{3}{*}{ Self-satisfaction } & I am very talented & 25.72 & 5.87 & \multirow{3}{*}{4.128} & \multirow{3}{*}{$0.017^{*}$} \\
\hline & I am quite talented & 23.74 & 5.51 & & \\
\hline & I am not very talented & 20.09 & 6.24 & & \\
\hline
\end{tabular}

Model; $\mathrm{F}=1.841, \mathrm{p}<0.05, \mathrm{R}^{2}=0.250$

$* \mathrm{p}<0.05$ significant effect, $\mathrm{p}>0.05$ no significant effect

The model established to determine the factors affecting the Verbal Expression of Students is statistically significant $(\mathrm{F}=1.841, \mathrm{p}<0.05)$ (see Table 12). Alcohol use in the family affects students' ability to Express Themselves Verbally $(\mathrm{F}=4.308, \mathrm{p}<0.05)$. Those whose families use alcohol have the highest level of Verbal Expression (24.42). The level of getting along with peers of students affects their Verbal Expression $(\mathrm{F}=6.342, \mathrm{p}<0.05)$. Verbal Expression perception level is the highest among those who get along very well with their friends (25.07). The level of self-satisfaction of students affects their ability of Verbal Expression $(\mathrm{F}=4.128, \mathrm{p}<0.05)$. Those who think that they are very talented have the highest level of Verbal Expression perception (25.72). 
Table 13. Factors that affect coping skills among the sub-dimensions of Emotional Management skills

\begin{tabular}{|c|c|c|c|c|c|}
\hline \multirow{2}{*}{ Model } & & \multicolumn{2}{|c|}{ Coping } & \multirow{2}{*}{$\mathrm{F}$} & \multirow{2}{*}{$\mathrm{p}$} \\
\hline & & Mean & Std.Deviation & & \\
\hline \multirow{2}{*}{ Smoking in the family } & $\mathrm{No}$ & 12.77 & 3.15 & \multirow{2}{*}{3.370} & \multirow{2}{*}{0.068} \\
\hline & Yes & 13.02 & 3.29 & & \\
\hline \multirow{4}{*}{ Alcohol in the family } & No & 12.94 & 3.25 & \multirow{4}{*}{1.233} & \multirow{4}{*}{0.268} \\
\hline & Yes & 12.60 & 3.01 & & \\
\hline & Several & 12.46 & 2.83 & & \\
\hline & None & 13.13 & 3.37 & & \\
\hline \multirow{3}{*}{ Friends smoking } & A few or less & 12.88 & 3.26 & \multirow{3}{*}{3.104} & \multirow{3}{*}{$0.047 *$} \\
\hline & Some & 12.97 & 3.08 & & \\
\hline & Majority & 12.78 & 3.22 & & \\
\hline \multirow{3}{*}{ Friends treating others badly } & None & 13.11 & 3.37 & \multirow{3}{*}{2.498} & \multirow{3}{*}{0.084} \\
\hline & Several & 12.96 & 2.81 & & \\
\hline & Several and more & 12.53 & 3.30 & & \\
\hline \multirow{3}{*}{ Getting along with peers } & Very good & 13.96 & 3.19 & \multirow{3}{*}{2.786} & \multirow{3}{*}{0.064} \\
\hline & Pretty good & 12.65 & 3.03 & & \\
\hline & Not very good & 11.62 & 3.48 & & \\
\hline \multirow{3}{*}{ Common sense level of daily problems } & Quite a lot & 13.32 & 3.35 & \multirow{3}{*}{1.955} & \multirow{3}{*}{0.144} \\
\hline & A little & 12.69 & 2.80 & & \\
\hline & Not much & 11.79 & 3.67 & & \\
\hline \multirow{3}{*}{ Feeling about opinions } & Very satisfied & 14.22 & 3.62 & \multirow{3}{*}{1.641} & \multirow{3}{*}{0.196} \\
\hline & Pretty satisfied & 12.91 & 3.11 & & \\
\hline & Not very satisfied & 12.10 & 2.88 & & \\
\hline \multirow{6}{*}{ Self-satisfaction } & I am very talented & 14.16 & 3.43 & \multirow{6}{*}{6.151} & \multirow{6}{*}{$0.002 *$} \\
\hline & I am quite talented & 12.84 & 2.87 & & \\
\hline & I am not very talented & 11.17 & 2.95 & & \\
\hline & Probably yes & 12.83 & 3.25 & & \\
\hline & Probably no & 13.40 & 2.84 & & \\
\hline & Occasionally and less & 12.14 & 2.99 & & \\
\hline \multirow{3}{*}{$\begin{array}{l}\text { Going to cinema, theater and attending } \\
\text { events with family }\end{array}$} & Always & 13.34 & 3.35 & \multirow{3}{*}{3.080} & \\
\hline & Frequently & 12.69 & 3.02 & & $0.048 *$ \\
\hline & Occasionally and less & 12.71 & 3.18 & & \\
\hline
\end{tabular}

Model; $\mathrm{F}=2.640, \mathrm{p}<0.05, \mathrm{R}^{2}=0.323$

$*_{\mathrm{p}}<0.05$ significant effect, $\mathrm{p}>0.05$ no significant effect

The model established to determine the factors affecting Coping of Students is statistically significant $(\mathrm{F}=2.640, \mathrm{p}<0.05)$ (see Table 13). Having smoker friends affects Coping $(\mathrm{F}=3.104, \mathrm{p}<0.05)$. Those with some smoker friends have the highest level of Coping perception (12.97). The level of self-satisfaction of students affects Coping $(\mathrm{F}=6.151, \mathrm{p}<0.05)$. Coping perceptions of those who consider themselves very talented are the highest (14.16). Going to the cinema and theater and participating in similar activities affects Coping $(\mathrm{F}=3.080, \mathrm{p}<0.05)$. Students who always go to the cinema and theater and participate in similar activities have the highest level of Coping perception (13.34). 
Table 14. Factors Affecting Students' Emotional Management Skills

\begin{tabular}{|c|c|c|c|c|c|}
\hline \multirow{2}{*}{ Model } & & \multicolumn{2}{|c|}{ Emotional Management } & \multirow{2}{*}{$\mathrm{F}$} & \multirow{2}{*}{$\mathrm{p}$} \\
\hline & & Mean & Std. Deviation & & \\
\hline \multirow{2}{*}{ Smoking in the family } & No & 91.75 & 12.64 & \multirow{2}{*}{0.352} & \multirow{2}{*}{0.554} \\
\hline & Yes & 93.45 & 12.99 & & \\
\hline \multirow{2}{*}{ Alcohol in the family } & No & 92.14 & 12.34 & \multirow{2}{*}{2.495} & \multirow{2}{*}{0.116} \\
\hline & Yes & 93.46 & 14.38 & & \\
\hline \multirow{3}{*}{ Smoker friends } & A few or less & 92.66 & 12.93 & \multirow{3}{*}{1.042} & \multirow{3}{*}{0.355} \\
\hline & Some & 93.62 & 12.11 & & \\
\hline & Majority & 91.15 & 13.24 & & \\
\hline \multirow{5}{*}{ Friends treating others badly } & None & 95.14 & 12.51 & \multirow{5}{*}{5.310} & \multirow{5}{*}{$0.006^{*}$} \\
\hline & Several & 91.62 & 11.28 & & \\
\hline & Several and more & 90.33 & 13.89 & & \\
\hline & Several & 91.10 & 11.17 & & \\
\hline & Several and more & 93.31 & 12.03 & & \\
\hline \multirow{3}{*}{ Getting along with peers } & Very good & 96.74 & 12.90 & \multirow{3}{*}{4.765} & \multirow{3}{*}{$0.009^{*}$} \\
\hline & Pretty good & 91.99 & 11.26 & & \\
\hline & Not very good & 85.67 & 15.97 & & \\
\hline \multirow{3}{*}{ Self-satisfaction } & I am very talented & 97.60 & 12.87 & \multirow{3}{*}{4.804} & \multirow{3}{*}{$0.009^{*}$} \\
\hline & I am quite talented & 92.90 & 11.17 & & \\
\hline & I am not very talented & 83.83 & 12.42 & & \\
\hline
\end{tabular}

Model; $\mathrm{F}=2.184, \mathrm{p}<0.05, \mathrm{R}^{2}=0.283$

$* \mathrm{p}<0.05$ significant effect, $\mathrm{p}>0.05$ no significant effect

The model established to determine the factors that affect students' Emotional Management Skills is statistically significant $(\mathrm{F}=2.184, \mathrm{p}<0.05)$ (see Table 14). The negative behaviors of students' friends towards others affect Emotional Management Skill $(\mathrm{F}=5.310, \mathrm{p}<0.05)$. Those with friends who do not behave badly to others have the highest level of Emotional Management Skill (95.14). The negative behaviors of students' friends towards others affect Emotional Management Skill $(\mathrm{F}=4.765, \mathrm{p}<0.05)$. Emotional Management Skills perception level is the highest among those who get along very well with their peers (96.74). The level of self-satisfaction of students affects Emotional Management Skills $(\mathrm{F}=4.804, \mathrm{p}<0.05)$. Those who find themselves very talented have the highest level of Emotional Management Skill perception (97.60).

\subsection{Logistic Regression Results}

In the analyses, smoking, drinking alcohol, doing something to harm the body, feeling unhappy and bad, and absenteeism were taken as reference.

Table 15. Reference Categories

\begin{tabular}{lll}
\hline Variable & Category & \multicolumn{1}{c}{ Reference } \\
\hline Smoking & Doesn't smoke & 0 \\
\cline { 2 - 3 } Alcohol & Smokes & 0 \\
\hline Self-harm & Doesn't drink & 1 \\
\cline { 2 - 3 } & Drinks & no \\
\cline { 2 - 3 } Unhappiness, feeling bad & yes & 1 \\
\cline { 2 - 3 } & no & 0 \\
\hline
\end{tabular}


Table 16. The Effects of Scale Scores on Smoking Status

\begin{tabular}{|c|c|c|c|c|c|c|}
\hline Dependent Var. & Independent Var. & Coefficient & sh. & Wald & $\mathrm{p}$ & ODDS ratio \\
\hline \multirow[t]{11}{*}{ Smoking } & Social Exclusion & -0.028 & 0.021 & 1.763 & 0.184 & 0.972 \\
\hline & Being ignored & 0.021 & 0.042 & 0.243 & 0.622 & 1.021 \\
\hline & Safety & -0.032 & 0.018 & 3.201 & 0.074 & 0.969 \\
\hline & Intimacy & 0.090 & 0.027 & 10.867 & $0.001 *$ & 1.094 \\
\hline & Acceptance & 0.004 & 0.043 & 0.010 & 0.922 & 1.004 \\
\hline & Help & -0.082 & 0.055 & 2.216 & 0.137 & 0.921 \\
\hline & Verbal Expression Ability & -0.010 & 0.024 & 0.177 & 0.674 & 0.990 \\
\hline & Showing Emotions as They Are & -0.032 & 0.030 & 1.149 & 0.284 & 0.968 \\
\hline & Ability to Control Negative & -0.035 & 0.036 & 0.957 & 0.328 & 0.966 \\
\hline & Coping & -0.044 & 0.038 & 1.343 & 0.247 & 0.957 \\
\hline & Anger Management & -0.070 & 0.056 & 1.561 & 0.212 & 0.932 \\
\hline
\end{tabular}

-2LL=489.643 Cox\&Snell R2=0.066, Nagelkerke R2=0.094

$\mathrm{X}^{2}=29.429, \mathrm{p}<0,05$

$* \mathrm{p}<0.05$ significant effect, $\mathrm{p}>0.05$ no significant effect

The established model is statistically significant $\left(\mathrm{X}^{2}=29.429, \mathrm{p}<0.05\right)$. Intimacy $(\mathrm{B}=0.090)$ positively affects smoking $(\mathrm{p}<0.05)$, (see Table 16).

Table 17. The Effects of Scale Scores on Alcohol Use

\begin{tabular}{|c|c|c|c|c|c|c|}
\hline Dependent Var. & Independent Var. & Coefficient & sh. & Wald & $\mathrm{p}$ & ODDS ratio \\
\hline \multirow{11}{*}{ Alcohol Use } & Social Exclusion & -0.052 & 0.023 & 5.128 & $0.024 *$ & 0.949 \\
\hline & Being ignored & 0.056 & 0.044 & 1.633 & 0.201 & 1.057 \\
\hline & Safety & -0.047 & 0.019 & 6.509 & $0.011 *$ & 0.954 \\
\hline & Intimacy & 0.096 & 0.029 & 11.379 & $0.001 *$ & 1.101 \\
\hline & Acceptance & -0.008 & 0.045 & 0.032 & 0.859 & 0.992 \\
\hline & Help & -0.102 & 0.057 & 3.150 & 0.076 & 0.903 \\
\hline & Verbal Expression Ability & 0.000 & 0.025 & 0.000 & 0.991 & 1.000 \\
\hline & Showing Emotions as They Are & -0.012 & 0.032 & 0.151 & 0.698 & 0.988 \\
\hline & Ability to Control Negative & -0.022 & 0.038 & 0.333 & 0.564 & 0.978 \\
\hline & Coping & -0.088 & 0.041 & 4.673 & $0.031 *$ & 0.915 \\
\hline & Anger Management & 0.044 & 0.060 & 0.531 & 0.466 & 1.045 \\
\hline \multicolumn{7}{|c|}{$-2 \mathrm{LL}=452.750$ Cox $\&$ Snell R2=0.068, Nagelkerke R2=0.101 } \\
\hline $\mathrm{X}^{2}=30.328$ & & & & & & \\
\hline
\end{tabular}

The established model is statistically significant $\left(X^{2}=30.328, \mathrm{p}<0.05\right)$. While Social Exclusion $(B=-0.052)$, Safety $(B=-0.047)$ and Coping $(B=0.088)$ had a negative effect on alcohol use, Intimacy had a positive effect $(p<0,05),($ see Table 17). 
Table 18. The Effects of Scale Scores on Feeling Bad, Unhappiness

\begin{tabular}{|c|c|c|c|c|c|c|}
\hline Dependent Var. & Independent Var. & Coefficient & sh. & Wald & $\mathrm{p}$ & ODDS ratio \\
\hline \multirow{11}{*}{$\begin{array}{l}\text { Feeling Bad, } \\
\text { Unhappiness }\end{array}$} & Social Exclusion & 0.009 & 0.020 & 0.215 & 0.643 & 1.009 \\
\hline & Being ignored & 0.094 & 0.039 & 5.719 & $0.017 *$ & 1.099 \\
\hline & Safety & -0.040 & 0.018 & 5.208 & $0.022 *$ & 0.961 \\
\hline & Intimacy & 0.051 & 0.026 & 3.874 & $0.049 *$ & 1.052 \\
\hline & Acceptance & -0.019 & 0.042 & 0.202 & 0.653 & 0.981 \\
\hline & Help & -0.010 & 0.054 & 0.032 & 0.859 & 0.990 \\
\hline & Verbal Expression Ability & 0.001 & 0.024 & 0.003 & 0.955 & 1.001 \\
\hline & Showing Emotions as They Are & -0.011 & 0.030 & 0.134 & 0.714 & 0.989 \\
\hline & Ability to Control Negative & -0.158 & 0.037 & 18.410 & $0.000^{*}$ & 0.854 \\
\hline & Coping & -0.084 & 0.039 & 4.672 & $0.031 *$ & 0.920 \\
\hline & Anger Management & 0.024 & 0.057 & 0.179 & 0.672 & 1.025 \\
\hline
\end{tabular}

-2LL=502.485 Cox\&Snell R2=0.142, Nagelkerke R2=0.194

$\mathrm{X}^{2}=66.097, \mathrm{p}<0.05$

$* \mathrm{p}<0.05$ significant effect, $\mathrm{p}>0.05$ no significant effect $=$

The established model is statistically significant $\left(X^{2}=66.097, p<0.05\right)$. Safety $(B=-0.040)$, Ability to control negative physical reactions $(\mathrm{B}=-0,158)$ and Coping $(\mathrm{B}=-0.084)$ affect feeling bad and unhappiness negatively, while Being neglected $(B=0.094)$ and Intimacy $(B=0.051)$ positively affect them $(\mathrm{p}<0.05)$, (see Table 18$)$.

Table 19. The Effects of Scale Scores on self Harm

\begin{tabular}{|c|c|c|c|c|c|c|}
\hline Dependent Variable & Independent Variable & Coefficient & sh. & Wald & $\mathrm{p}$ & ODDS ratio \\
\hline \multirow{11}{*}{ Self harm } & Social Exclusion & -0.007 & 0.022 & 0.091 & 0.763 & .993 \\
\hline & Being ignored & 0.048 & 0.042 & 1.354 & 0.245 & 1.050 \\
\hline & Safety & -0.051 & 0.019 & 7.463 & $0.006^{*}$ & 0.950 \\
\hline & Intimacy & 0.039 & 0.027 & 2.048 & 0.152 & 1.040 \\
\hline & Acceptance & -0.024 & 0.045 & 0.294 & 0.588 & 0.976 \\
\hline & Help & -0.002 & 0.058 & 0.001 & 0.975 & 0.998 \\
\hline & Verbal Expression Ability & 0.026 & 0.025 & 1.014 & 0.314 & 1.026 \\
\hline & Showing Emotions as They Are & -0.028 & 0.032 & 0.760 & 0.383 & 0.973 \\
\hline & Ability to Control Negative Physical & -0.105 & 0.039 & 7.346 & $0.007 *$ & 0.900 \\
\hline & Coping & -0.037 & 0.041 & 0.810 & 0.368 & 0.963 \\
\hline & Anger Management & 0.016 & 0.062 & 0.069 & 0.792 & 1.016 \\
\hline
\end{tabular}

-2LL=443.270 Cox\&Snell R2=0.059, Nagelkerke R2=0.088

$\mathrm{X}^{2}=26.060, \mathrm{p}<0.05$

$* \mathrm{p}<0.05$ significant effect, $\mathrm{p}>0.05$ no significant effect

The established model is statistically significant $\left(X^{2}=26.060, p<0.05\right)$. Safety $(B=-0.051)$ and Ability to control negative physical reactions $(B=-0.105)$ have a negative effect on self-harm $(p<0.05)$, (see Table 19). 
Table 20. The Effects of Scale Total Scores on Feeling Bad, Unhappiness

\begin{tabular}{llcccccc}
\hline Dependent Var. & Independent Var. & Coefficient & sh. & Wald & p & ODDS ratio \\
\hline \multirow{3}{*}{$\begin{array}{l}\text { Feeling Bad, } \\
\text { Unhappiness }\end{array}$} & Friendship Quality & -0.008 & 0.006 & 2.094 & 0.148 & 0.992 \\
\cline { 2 - 7 } & Need to Belong & 0.008 & 0.019 & 0.157 & 0.691 & 1.008 \\
\cline { 2 - 7 } & Social Competence & 0.030 & 0.024 & 1.489 & 0.222 & 1.030 \\
\cline { 2 - 7 } & Emotional Management Skills & -0.045 & 0.010 & 22.523 & $0.000^{*}$ & 0.956 \\
\hline
\end{tabular}

-2LL=535.760 Cox\&Snell R2=0.073, Nagelkerke R2=0.100

$\mathrm{X}^{2}=32.821, \mathrm{p}<0.05$

$* \mathrm{p}<0.05$ significant effect, $\mathrm{p}>0.05$ no significant effect

The established model is statistically significant $\left(X^{2}=32.821, p<0.05\right)$. Emotional Management Skills $(B=-0.045)$ affect feeling bad and unhappiness negatively $(\mathrm{p}<0.05)$, ( see table 20$)$.

Table 21. The Effects of Scale Total Scores on Self Harm

\begin{tabular}{llccccc}
\hline Dependent Var. & Independent Var. & Coefficient & sh. & Wald & $\mathrm{p}$ & ODDS ratio \\
\hline \multirow{3}{*}{ Self harm } & Friendship Quality & -0.004 & 0.006 & .468 & 0.494 & 0.996 \\
\cline { 2 - 7 } & Need to Belong & -0.012 & 0.022 & .308 & 0.579 & 0.988 \\
\cline { 2 - 7 } & Social Competence & -0.035 & 0.026 & 1.859 & 0.173 & 0.965 \\
\cline { 2 - 7 } & Emotional Management Skills & -0.020 & 0.010 & 3.813 & $0.050^{*}$ & 0.981 \\
\hline
\end{tabular}

-2LL=458.155 Cox\&Snell R2=0.026, Nagelkerke R2=0.039

$\mathrm{X}^{2}=11.175, \mathrm{p}<0.05$

$* \mathrm{p}<0.05$ significant effect, $\mathrm{p}>0.05$ no significant effect

The established model is statistically significant $\left(\mathrm{X}^{2}=11.175, \mathrm{p}<0.05\right)$. Emotional Management Skills $(\mathrm{B}=-0.020)$ negatively affect body damage $(\mathrm{p}<0.05)$, (see Table 21$)$.

\section{Discussion}

When the findings of the research are evaluated in general, it can be deduced that social exclusion, friendship quality and emotional management skills can be protective factors for the problem behaviors of young people, and it is seen that the findings support the relevant literature and research findings.

Peer relations constitute almost all of the daily social relationships of adolescents. For adolescents, social exclusion means being left alone by their peers and unintentionally being pushed out of the group. This gives information regarding that they are not sufficient in terms of the characteristics defined by their peers. The findings of the study emphasize the competencies that young people should have to be present in peer groups. The findings regarding social exclusion reveal that the same characteristics may lead to different but similar levels of exclusion (Peplak, Song, Colasante, Malti, 2017) (Paget, Parker, Heron, Logan, HenleyEmond, Ford, 2018). These characteristics include having smoker friends, that others behave badly and negatively, disagreement with their peers and dissatisfaction with their physical appearance. It is seen that being sensitive to and interested in social events increase the perception of exclusion in other dimensions except the sub-dimension of being cared. The findings related to social exclusion and its sub-dimensions are in line with the literature on risk behaviors and problem behaviors in adolescents. The studies conducted on smoking, alcohol and substance use consider activities related to these as risk behaviors or problem behaviors (Kobus, 2003; Leung, Toumbourou, Hemphill, 2014).Even though adolescents can consciously prefer smoking as a risk behavior to mimick adult role model behaviors or prove their own competence, this behavior should bring short-term benefit. According to the problem-behavior theory (Jessor, 2014), adolescents experience developmental behavior and adaptation problems due to the insufficiency of protective factors, presence of triggering factors, find it difficult to produce effective solutions and may feel as if they are able to solve exogenous behaviors such as cigarette and alcohol use, which are not healthy ways of coping. Therefore, it provides information on the lack of effective solutions to problems encountered in other areas of life such as smoking and alcohol and substance use. In other words, the three factors that increase social exclusion can be the causes and consequences of each other. Studies show that body perception affects interpersonal relationships among developmental adaptation problems in adolescents, in that, young people who do not have positive thoughts and feelings about their physical appearance have low self-esteem, self-efficacy and self-worth, which has negative consequences for initiating and sustaining friendship 
relationships (Guilamo-Ramos, Litardo, Jaccard, 2005; Meilstrup, Thygesen, Nielsen, 2016). In addition, considering the finding that the sensitivity to social issues is a factor affecting exclusion and the finding that this is one of the factors that increase individuals' perception of their social competence, the schemes on the basis of thoughts, emotions and behaviors related to individuals expressing their thoughts become more important during the socialization process. The knowledge gained by adolescents' friends, who exhibit these behaviors, through the educational environment and how they are perceived in the family and peer circles is important (Rosenbloom, 2003). In other words, it is necessary to answer the question for whom, what characteristic means what, and risk behavior and problem behavior theory in adolescents answer these questions from a different perspective (Leather, 2009). In addition, the perception of themselves and their friends on social issues, Adler's views on the impact of contrast and comparison behaviors of the social environment on the individual and Ericson's attention to the dynamics of children's socialization process in pre-adolescence period evoke the effect of feelings of inferiority and insufficiency compared to success in these dynamics (Lease, Musgrove, Axelrod, 2002). As Maslow puts it in his theory of hierarchy of needs (Greenhalgh, Wessely, 2004; Miller, Miller, 2009), one of the fundamental needs of the individual is respectability and a sense of accomplishment. It is thought that the opinions of young people about themselves and others and their relationships developing accordingly are also important for academic success.

The findings suggest that, even if it appears to be in contradiction with social exclusion in the dimension of smoking, the question of who thinks smoking increases the feeling of intimacy among friends should be answered. The researches on adolescents show that adolescents are members of the same groups that are like them and have similar problems (Kobus, 2003). This reveals a phenomenon not only seen in adolescent behavior but also in adult behavior: "People feel better with others who can empathize with themselves." They avoid relationships where there are such communication elements as judgment, criticism, guidance and inadequacy (Bagwell, Bukowski, 2018). One of the basic needs of people is to be accepted as they are. From a different point of view, we can find information that can support this idea in another factor that affects the feeling of intimacy: "Those who have a good understanding with their friends have a higher perception of intimacy." In addition, this finding is in line with the findings on the factors affecting social competence and social exclusion, and the highest level of social competence perception among the individuals who have smokers in the family and who have a good understanding with their peers. On the other hand, as a factor increasing the perception of social competence, the finding that there is a smoker in the family points out that the factors leading to health-threatening behaviors should be evaluated in a multidimensional way (Nam, Fukui, 2017). Many factors need to be considered, including family functions, the solutions the parent produces through these behaviors or the role of the parent as a means of identification in family or social relationships or the behaviors learnt by the adolescent as criteria of reaching adulthood (Segrin, Flora, 2011). One of the factors that affect intimacy is related to the economic status of the family of adolescent (Freund, Lous, 2012). The students who stated that they had some or less economic difficulties with their parents had the highest perception of intimacy. This situation can be evaluated with the phenomenon of inequality that humanity has reached to the information society from an interdisciplinary point of view but still does not produce a solution (Plenty, Mood, 2016; Lorant, Rojas, 2017). Therefore, it is natural that individuals who have basic life resources can reach them more easily or face less difficulties related to them can feel stronger in all areas of life, as in social relations. In other words, although it is stated that caste systems existed centuries ago and education provides the opportunity to move up and down the social ladder, the findings obtained here and interdisciplinary research findings show that this cannot be overcome, that is, having a certain economic quality of life or being less distressed about it makes it easier to maintain social and close relationships.

It is seen that the findings that having family members using alcohol perceive themselves more than sufficient in terms of expressing themselves verbally (Kogan, 2017) and that those with smoker family members have the highest perception of social competency are in parallel with each other (Lee, Cranford, 2008). In addition, this is consistent with the finding that students who state that they had some smoker friends had the highest problem-solving competence in terms of emotional management skills and problem-solving dimension. From a psychoanalytic perspective, it is possible to say that alcohol, etc. has a kind of mediation role in expressing experiences that cannot be expressed in consciousness in everyday life, which create a sense of frustration and are pushed into the unconscious (Wang, Liu, Zhan, Shi, 2010). The literature on risk-taking behavior correlates adolescents' use of substances such as cigarettes, alcohol, etc., as well as their orientation towards other risk behaviors with dynamics such as being able to perform adulthood behaviors, proving oneself, satisfying the need for short-term relaxation and the belief that they can control the outcomes of their behaviors or their own tendencies (Richter, 2010). The perspective of social learning theory, on the other hand, acts as the indirect reinforcer role of behaviors where another is rewarded. Therefore, the effect of alcohol use by family members can be encouraging for young people, and research emphasize that proximity to cigarettes, alcohol and drugs can play a triggering role in the use of these substances (Hill, Chow, 2002). It is seen that those who can communicate well with their friends, who find themselves capable enough and capable of expressing themselves verbally and coping with problems with their emotion management skills and sub-dimensions are found to have the highest perceptions; the 
findings are in parallel with findings on social exclusion, friendship quality and social competence. In addition, the perceptions of young people who stated that they had no friends who behaved badly to others had the highest perceptions of their ability to manage their emotions. Therefore, as the relevant literature emphasizes, peer relationships have undeniable importance in adolescents' lives (Klein, Cornell, Konold, 2012; Corsano, Musetti, Caricati, Magnani, 2017).

The finding that those who can participate in activities such as cinema, theater, etc. with their family have the highest perceptions about their ability to cope with problems correlates with the finding that those who have some or less economic problems in their family have the highest perception of intimacy (Berg, Kiviruusu, Karvonen, Rahkonen, Huurre, 2017). According to World Health Organization quality standards of life, participation in such activities is one of the basic human needs and can also contribute to the socialization process. Considering that visual arts such as theater and cinema are the means of telling mankind's own stories to each other, it is possible to state that they can teach not only the sense of empathy but also the ability to manage problem-solving.

One of the main aims of the study is to determine the prediction levels of adolescents' social exclusion, friendship quality, social competence and emotional management skills on risk behaviors according to risk-taking behavior theory, externalized behavior and adaptation problems according to problem behavior theory, smoking, alcohol use and unhappiness, hopelessness, feeling bad and self-harm (bodily-harm) within the internal problem behavior category. Logistic regression analysis results support the other findings of the study in this regard (Leary, 1990 ;Hasking, Lyvers, Carlopio, 2011; Tomé, de Matos, Simões, Camacho, AlvesDiniz, 2012; Gioia, 2017; Lodder, Scholte, Goossens, Verhagen, 2017; Mason, Zaharakis, Rusby, Westling, Light, Mennis, Flay, 2017).

The findings indicate that the quality of friendship intimacy is effective on smoking behavior. The intimacy of the quality of friendship reveals that perceiving and understanding the support and friendship of their friends increases smoking (Janssens, Van Den Noortgate, Goossens, Verschueren, Colpin, Claes, Van Leeuwen, 2017). The finding is consistent with the findings on the factors affecting the intimacy dimension. Considering the factors that affect the social exclusion characteristics of the participants, the fact that social exclusion has no effect on smoking is perceived as a natural result to some extent and that young people are relieved by their friends' problem-solving behaviors not to be excluded by them or as a social psychological phenomenon, there may be individuals with similar problems, problem-solving behaviors or tendencies. Considering the increasing effect of the related dimension of friendship quality on alcohol use and smoking, it becomes evident that the characteristics of young people and their group of friends, family, school and personality characteristics should be examined. Therefore, it is evident that the characteristics of the individual and social environment that determine behaviors should be evaluated as a whole (Marsden, Boys, FarrellStillwell Hutchings, Hillebrand, Griffiths, 2005).The findings of the study on coping with problems of social security, social exclusion and emotional management skills have a reducing effect on alcohol use support this idea. Considering that emotional management skills are closely related to the competences of social support resources gained through individual experience, especially the family functions, are an important factor. The security dimension emphasizes that one cannot be harmed by the existence of other people, as Maslow's hierarchy of needs emphasizes trust, and Ericson's emphasis on infancy regarding distrust versus basic trust. It is seen that the security dimension of the quality of friendship plays a protective role on unhappiness, hopeless, bad feeling and self-harm (bodily harm), as in alcohol use. That is, the security dimension has a negative, reducing effect on negative mood and self-harm (Heilbron, Prinstein, 2008; Prinstein, Heilbron, Guerry, 2011). Research indicates that self-harm is more common in cases of abuse and neglect. Self-harm is a kind of impulsive, repetitive, non-lethal or non-suicidal kind of help where individuals indicate that they cannot get themselves out of doing such behavior as a kind of unhealthy coping skill in the face of problems (Taliaferro, Muehlenkamp, Borowsky, McMorris, Kugler, 2012). Especially traumatic events, depending on the traumatic behavior experienced, not being able to prevent this experience creates feelings of guilt and self-punishment for the individual; the body feels pain and pleasure, relaxation and power; so, the behavior is performed with conflicting thoughts and emotions. The relevant literature shows that all types of abuse and neglect can increase the tendency to perform such behaviors and that young people may be affected by other individuals showing similar behaviors to solve their problems (Hallab, Covic, 2010; Adrian, Zeman, Erdley, 2011; Peh, Shahwan, Fauziana, Mahesh, Sambasivam, Zhang,..., Subramaniam, 2017). Therefore, the security dimension draws attention to the reductive effect of knowing the existence of friends who can direct the youth to healthy behaviors and provide the health support they need (Barry,Lui, Lee-Rowland, Moran, 2017; Schwartz-Mette, Smith, 2018).

The findings on depressive affect, feeling unhappy and hopeless, the intimacy dimension and the positive, enhancing effect of caring dimensions explaining social exclusion by friends support other findings and related literature (Schwartz-Mette, Smith, 2018; Lorant, Tranmer, 2019). Mobbing in the business world is seen as peer bullying in peer relationships and bullying in love affairs or personal relationships (Siyez, M., Aysan, 2007; Vanderbilt, Augustyn, 2010). It is seen that psycho-social risk predisposition is one of the determinants of young people who will not be 
harmed and can establish themselves with their peers with whom they feel safe (Kochel, Bagwell, Ladd, Rudolph, 2017).

As in the other findings on emotional management skills, it was found that emotional management skills act as a protective factor (Carthy, Horesh, Apter, Gross, 2010; Yeager, 2017) in self-harm and depressive affect, that is, internalized problem behaviors. This finding may provide effective solutions to "sudden emotional changes" that are perceived as a characteristic feature of adolescence and can be ignored by adolescents' families, especially teachers, and other adults as temporary or to which no one feels sufficient enough to react. As a result, in terms of social exclusion, friendship quality and emotional management skills and social competence, the study investigated risk-taking behaviors and problem behavior theory as well as behavior and adaptation problems, and it is consistent with the research findings highlighting the characteristics of adolescents that can be determined by the susceptibility of the psycho-social risk tendency.

\section{Conclusion and Suggestions}

This study was conducted to determine the predictive effect of the features (scale scores) related the factors that affect social exclusion, friendship quality, social competence and emotional management skills in adolescents on adolescent problem behaviors such as smoking, alcohol use, unhappiness, hopelessness and self-harm behaviors. The study reveals that the related factors can be either a protective or triggering factor that can determine the psychosocial risk predisposition of adolescents. According to the findings on social exclusion, those who have smoker friends, those with friends who treat others negatively, those who do not get along with their peers very well, those who have negative perceptions of their appearance and those who are interested in social events have a higher perception of social exclusion. Social exclusion was found to have a reducing effect on alcohol use. It was found that social competence was influenced by the presence of a smoker in the family, a very good relationship with their peers, an interest in social events, and no predictive effect on problem behaviors was determined. According to the findings on the factors affecting the quality of friendship, it was found that the intimacy dimension was influenced by having mostly smoker friends, getting along with peers very well and experiencing economic distress in the family to some extent or less frequently. It was determined that friendship quality and intimacy had an increasing effect on alcohol use and negative moods, and the security dimension had a decreasing effect on alcohol use, negative mood and self-harm.

In verbal self-expression, alcohol use in the family, getting along well with friends, having smoker friends, perceiving oneself as very skilled, family activities such as cinema, theater, etc., having no friends who behave badly to others, getting along with peers very well and perceiving oneself as very talented made a difference. Emotional management skills and coping with problems had a diminishing effect on alcohol use, and negative mood (unhappiness-hopelessness) and ability to control negative physical reactions had a reducing effect on self-harm.

The results showed the importance of peer relationships and the ability of adolescents to control emotions that make management of interpersonal relationships difficult for adolescents to solve their behavior and adaptation problems.

This study was designed to contribute to the prevention of self-harm behaviors, which are seen more frequently in cases of abuse and neglect and increase smoking and alcohol use. The findings support the related literature and show the importance of emotional management skills and friendship relationships in the life of adolescents, as in adult life. Therefore, it is considered that studies to be conducted on the management of emotional processes of adolescents should address not only adolescents, but also families and educators. In particular, it is necessary to raise awareness that sudden changes in emotions, which are accepted as characteristic of adolescence and somehow normalized, can affect their coping process. Emotional changes in adolescents are also a reflection of the support they need, and it is thought that studies aimed at restructuring adults' own roles and functions, especially family, are of utmost importance. It is necessary to raise awareness regarding the fact that adults are responsible for the behaviors of the young people who have positive behaviors and praise and perceive the group as negative and problematic. In other words, it is thought that the social environment of adolescents should be made aware of how they are role models for young people in their dynamics regarding emotional management, choice of friends and maintenance of friendship relationships in all areas of life.

\section{References}

Adrian, M., Zeman, J., Erdley, C., Lisa, L., \& Sim, L. (2011). Emotional Dysregulation and Interpersonal Difficulties as Risk Factors for Nonsuicidal Self-Injury in Adolescent Girls, J. Abnorm Child Psychol, 39, 389. https://doi.org/10.1007/s10802-010-9465-3

Agresti, A. (1996). An introduction to categorical data analysis, JohnWiley \& Sons. Inc., Publication.

Akın, A., Doğan, R., Gönülalan, G. D., Atik, R., Çebiş, T., \& Akın, U. (2014). Ergenler için sosyal dışlanma ölçeği Türkçe formunun geçerlik ve güvenirliği. Paper presented at the $1^{s t}$ Eurasian Educational Research Congress, 
April, 24-26, İstanbul, Türkiye.

Akın, A., Karduz Adam, F. F., \& Akın, Ü. (2014). Arkadaşlık kalitesi ölçeği Türkçe formunun geçerlik ve güvenirliği. Journal of Research in Education and Teaching, 3(4), 378-383. https://doi.org/10.14686/BUEFAD.201428191

Anderson, S. L., \& Betz, N. E. (2001). Sources of social self-efficacy expectations: Their measurement and relation to career development. Journal of Vocational Behaviour, 58(1), 98-117. https://doi.org/10.1006/jvbe.2000.1753

Archer, K. J., Lemeshow, S., \& Hosmer, D. W. (2007). Goodness-of-fit tests for logistic regression models when data are collected using a complex sampling design. Computational Statistics \& Data Analysis, 51(9), 4450-4464. https://doi.org/10.1016/j.csda.2006.07.006

Bagwell, C. L., \& Bukowski, W. M. (2018). Friendship in childhood and adolescence: Features, effects, and processes. In W. M. Bukowski, B. Laursen, \& K. H. Rubin (Eds.), Handbook of peer interactions, relationships, and groups (pp. 371-390). New York, NY, US: The Guilford Press.

Barry, C. T., Lui, J. H., Lee-Rowland, L. M., \& Moran, E. V. (2017). Adolescent communal narcissism and peer perceptions. Journal of personality, 85(6), 782-792. https://doi.org/10.1111/jopy.12287

Berg, N., Kiviruusu, O., Karvonen, S., Rahkonen, O., \& Huurre, T. (2017). Pathways from poor family relationships in adolescence to economic adversity in mid-adulthood. Advances in Life Course Research, 32, 65-78. https://doi.org/10.1016/j.alcr.2016.07.001

Brody, G. H. (1998). Sibling relationship quality: Its causes and consequences. Annual Review of Psychology, 49(1), 1-24. https://doi.org/10.1146/annurev.psych.49.1.1

Brody, G. H. (2004). Siblings' direct and indirect contributions to child development. Current Directions in Psychological Science, 13(3), 124-126. https://doi.org/10.1111/j.0963-7214.2004.00289.x

Brown, B. (2004). Adolescents' relationships with peers. Richard M. Lerner and Laurence Steinberg (eds.) Handbook of adolescent psychologhy (363-394), (second Edition). New Jersey: John Wiley\&Sons, Inc. https://doi.org/10.1002/9780471726746.ch12

Brown, B. B., \& Klute, C. (2006). Friendships, cliques and crowds. Gerald R. Adams and Micheal D. Berzonsky (Eds.) Blackwell handbook of adolescence (330-349). UK: Blackwell Publishing. https://doi.org/10.1002/9780470756607.ch16

Bukowski, W. M., Newcomb, A. F., \& Hartup, W. W. (1996). Friendship and its significance in childhood and adolescence: Introduction and comment. The company they keep: Friendship in childhood and adolescence, 1-15.

Carlo, G., Crockett, L. J., Wolff, J. M., \& Beal, S. J. (2012). The role of emotional reactivity, self-regulation, and puberty in adolescents' prosocial behaviors. Social Development, 21(4), 667-685. https://doi.org/10.1111/j.1467-9507.2012.00660.x

Carthy, T., Horesh, N., Apter, A., \& Gross, J. J. (2010). Patterns of emotional reactivity and regulation in children with anxiety disorders. Journal of Psychopathology and Behavioral Assessment, 32(1), 23-36. https://doi.org/10.1007/s10862-009-9167-8

Çeçen, A. R. (2006). Duyguları yönetme becerileri ölçeğinin geliştirilmesi: Geçerlik ve güvenirlik çalışmaları. Türk Psikolojik Danışma ve Rehberlik Dergisi, 3(26), 101-113.

Collins, W. A., \& Laursen, B. (2004). Changing relationships, changing youth: Interpersonal contexts of adolescent development. The Journal of Early Adolescence, 24(1), 55-62. https://doi.org/10.1177/0272431603260882

Connolly, J. (1989). Social self-efficacy in adolescence: Relations with self-concept, social adjustment, and mental health. Canadian Journal of Behavioural Science/Revue Canadienne des Sciences du Ccomportement, 21 (3), 258. https://doi.org/10.1037/h0079809

Corsano, P., Musetti, A., Caricati, L., \& Magnani, B. (2017). Keeping secrets from friends: Exploring the effects of friendship quality, loneliness and self-esteem on secrecy. Journal of Adolescence, 58, 24-32. https://doi.org/10.1016/j.adolescence.2017.04.010

Duncan, S. C., Duncan, T. E., \& Strycker, L. A. (2000). Risk and protective factors influencing adolescent problem behavior: A multivariate latent growth curve analysis. Annals of Behavioral Medicine, 22(2), 103-109. https://doi.org/10.1007/BF02895772

Dusek, J. B., \& Julie, G. M. (2006). Self-concept and self-esteem development. Gerald R. Adams and Micheal D. Berzonsky (Eds.) Blackwell handbook of adolescence (290-300). UK: Blackwell Publishing. https://doi.org/10.1002/9780470756607.ch14 
Flynn, H. K., Felmlee, D. H., \& Conger, R. D. (2017). The social context of adolescent friendships: Parents, peers, and romantic partners. Youth \& Society, 49(5), 679-705. https://doi.org/10.1177/0044118X14559900

Freund, K. S., \& Lous, J. (2012). The effect of preventive consultations on young adults with psychosocial problems: a randomized trial. Health Education Research, 27(5), 927-945. https://doi.org/10.1093/her/cys048

Galambos, N. L., \& Costigan, C. L. (2003). Emotional and personality development in adolescence. Handbook of Psychology, 351-372. https://doi.org/10.1002/0471264385.wei0614

Gioia, F. (2017). Peer effects on risk behaviour: the importance of group identity. Experimental economics, 20(1), 100-129. https://doi.org/10.1007/s10683-016-9478-z

Good, M., Grand, M. P., Newby-Clark, I. R., \& Adams, G. R. (2008). The moderating effect of identity style on the relation between adolescent problem behavior and quality of psychological functioning. Identity: An International Journal of Theory and Research, 8(3), 221-248. https://doi.org/10.1080/15283480802181859

Greenhalgh, T., \& Wessely, S. (2004). 'Health for me': a sociocultural analysis of healthism in the middle classes. British Medical Bulletin, 69(1), 197-213. https://doi.org/10.1093/bmb/ldh013

Guerra, N. G., \& Bradshaw, C. P. (2008). Linking the prevention of problem behaviors and positive youth development: Core competencies for positive youth development and risk prevention. New directions for child and adolescent development, 2008(122), 1-17. https://doi.org/10.1002/cd.225

Gullone, E., \& Moore, S. (2000). Adolescent risk-taking and the five-factor model of personality. Journal of Adolescence, 23(4), 393-407. https://doi.org/10.1006/jado.2000.0327

Hallab, L., \& Covic, T. (2010). Deliberate self-harm: The interplay between attachment and stress. Behaviour Change, 27(2), 93-103. https://doi.org/10.1375/bech.27.2.93

Harris, K. M., Duncan, G. J., \& Boisjoly, J. (2002). Evaluating the role of "nothing to lose" attitudes on risky behavior in adolescence. Social Forces, 80(3), 1005-1039. https://doi.org/10.1353/sof.2002.0008

Hasking, P., Lyvers, M., \& Carlopio, C. (2011). The relationship between coping strategies, alcohol expectancies, drinking motives and drinking behaviour. Addictive behaviors, 36(5), 479-487. https://doi.org/10.1016/j.addbeh.2011.01.014

Heilbron, N., \& Prinstein, M. J. (2008). Peer influence and adolescent nonsuicidal self-injury: A theoretical review of mechanisms and moderators. Applied and Preventive Psychology, 12(4), 169-177. https://doi.org/10.1016/j.appsy.2008.05.004

Hill, E. M., \& Chow, K. (2002). Life-history theory and risky drinking. Addiction, 97(4), 401-413. https://doi.org/10.1046/j.1360-0443.2002.00020.x

Hosmer Jr, D. W., Lemeshow, S., \& May, S. (2008). Applied survival analysis: regression modeling of time-to-event data (Vol. 618). Wiley-Interscience. https://doi.org/10.1002/9780470258019

Janssens, A., Van Den Noortgate, W., Goossens, L., Verschueren, K., Colpin, H., Claes, S., .. Van Leeuwen, K. (2017). Adolescent externalizing behaviour, psychological control, and peer rejection: Transactional links and dopaminergic moderation. British journal of developmental psychology, 35(3), 420-438. https://doi.org/10.1111/bjdp.12184

Jessor, R. (1984). Adolescent development and behavioral health. Behavioral health: A Handbook of Health Enhancement and Disease Prevention, 69-90.

Jessor, R. (1987). Problem-behavior theory, psychosocial development, and adolescent problem drinking. British journal of addiction, 82(4), 331-342. https://doi.org/10.1111/j.1360-0443.1987.tb01490.x

Jessor, R. (2001). Problem-behavior theory. In Risikoverhaltensweisen Jugendlicher (pp. 61-78). https://doi.org/10.1007/978-3-663-11310-2_3

Jessor, R. (2014). Problem behavior theory: A half-century of research on adolescent behavior and development. Journal of Research on adolescence, 20(2), 274-286.

Kalaycı, Ş. (2006). SPSS uygulamalı çok değişkenli istatistik teknikleri (SPSS applied multivariate statistical techniques). Asil Yayın Dağıtım. Ankara, Turkey

Klein, J., Cornell, D., \& Konold, T. (2012). Relationships between bullying, school climate, and student risk behaviors. School Psychology Quarterly, 27(3), 154. https://doi.org/10.1037/a0029350

Kobus, K. (2003). Peers and adolescent smoking. Addiction, 98, 37-55. https://doi.org/10.1046/j.1360-0443.98.s1.4.x 
Kochel, K. P., Bagwell, C. L., Ladd, G. W., \& Rudolph, K. D. (2017). Do positive peer relations mitigate transactions between depressive symptoms and peer victimization in adolescence? Journal of applied developmental psychology, 51, 44-54. https://doi.org/10.1016/j.appdev.2017.04.003

Kogan, S. M. (2017). The role of parents and families in preventing young adult alcohol use. Journal of Adolescent Health, 61(2), 127-128. https://doi.org/10.1016/j.jadohealth.2017.05.019

Konrad, K., Firk, C., \& Uhlhaas, P. J. (2013). Brain development during adolescence: neuroscientific insights into this developmental period. Deutsches Ärzteblatt International, 110(25), 425. https://doi.org/10.3238/arztebl.2013.0425

Kuther, T. L. (2002). Rational decision perspectives on alcohol consumption by youth: Revising the theory of planned behavior. Addictive behaviors, 27(1), 35-47. https://doi.org/10.1016/S0306-4603(00)00161-1

Leahy, R. L. (2001). The construction of the self: A developmental perspective. Journal of Cognitive Psychotherapy, 15(4), 383. https://doi.org/10.1891/0889-8391.15.4.383

Leary, M. R. (1990). Responses to social exclusion: Social anxiety, jealousy, loneliness, depression, and low self-esteem. Journal of Social and Clinical Psychology, 9(2), 221-229. https://doi.org/10.1521/jscp.1990.9.2.221

Lease, A. M., Musgrove, K. T., \& Axelrod, J. L. (2002). Dimensions of social status in preadolescent peer groups: Likability, perceived popularity, and social dominance. Social Development, 11(4), 508-533. https://doi.org/10.1111/1467-9507.00213

Leather, N. C. (2009). Risk-taking behaviour in adolescence: a literature review. Journal of Child Health Care, 13(3), 295-304. https://doi.org/10.1177/1367493509337443

Lee, H. H., \& Cranford, J. A. (2008). Does resilience moderate the associations between parental problem drinking and adolescents' internalizing and externalizing behaviors?: A study of Korean adolescents. Drug and alcohol Dependence, 96(3), 213-221. https://doi.org/10.1016/j.drugalcdep.2008.03.007

Lerner, R. M., \& Steinberg, L. (2004). The scientific study of adolescent development. Handbook Of Adolescent Psychology, 2, 1-12. https://doi.org/10.1002/9780471726746

Leung, R. K., Toumbourou, J. W., \& Hemphill, S. A. (2014). The effect of peer influence and selection processes on adolescent alcohol use: a systematic review of longitudinal studies. Health psychology review, 8(4), 426-457. https://doi.org/10.1080/17437199.2011.587961

Lodder, G. M., Scholte, R. H., Goossens, L., \& Verhagen, M. (2017). Loneliness in early adolescence: Friendship quantity, friendship quality, and dyadic processes. Journal of Clinical Child \& Adolescent Psychology, 46(5), 709-720. https://doi.org/10.1080/15374416.2015.1070352

Lorant, V., \& Tranmer, M. (2019). Peer, school, and country variations in adolescents' health behaviour: A multilevel analysis of binary response variables in six European cities. Social Networks, 59, 31-40. https://doi.org/10.1016/j.socnet.2019.05.004

Lorant, V., Rojas, V. S., Robert, P. O. Kinnunen, J. M., Kuipers, M. A. G., Moor, I., ... Kunst, A. E. Social network and inequalities in smoking amongst school-aged adolescents in six European countries, Int. J. Public Health, 62(1), 53. https://doi.org/10.1007/s00038-016-0830-z

Malatesta, C. Z. (1990, January). The role of emotions in the development and organization of personality. In Nebraska symposium on motivation (Vol. 36, pp. 1-56).

Marsden, J., Boys, A., Farrell, M., Stillwell, G., Hutchings, K., Hillebrand, J., \& Griffiths, P. (2005). Personal and social correlates of alcohol consumption among mid-adolescents. British Journal of Developmental Psychology, 23(3), 427-450. https://doi.org/10.1348/026151005X26020

Mason, M. J., Zaharakis, N. M., Rusby, J. C., Westling, E., Light, J. M., Mennis, J., \& Flay, B. R. (2017). A longitudinal study predicting adolescent tobacco, alcohol, and cannabis use by behavioral characteristics of close friends. Psychology of Addictive Behaviors, 31(6), 712-720. https://doi.org/10.1037/adb0000299

McLaughlin, K. A., Megan, C. G., \& Leah, H. S. (2015). What develops during emotional development? A component process approach to identifying sources of psychopathology risk in adolescence. Dialogues in Clinical Neuroscience, 17(4), 403-410.

Meilstrup, C., Thygesen, L. C., Nielsen, L., Koushede, V., Cross, D., \& Holstein, B. E. (2016). Does self-efficacy mediate the association between socioeconomic background and emotional symptoms among schoolchildren, Int. J. Public Health, 61(4), 505. https://doi.org/10.1007/s00038-016-0790-3

Meuwese, R., Cillessen, A. H., \& Güroğlu, B. (2017). Friends in high places: A dyadic perspective on peer status as 
predictor of friendship quality and the mediating role of empathy and prosocial behavior. Social Development, 26(3), 503-519. https://doi.org/10.1111/sode.12213

Miller, P. G., \& Miller, W. R. (2009). What should we be aiming for in the treatment of addiction? Addiction, 104(5), 685-686. https://doi.org/10.1111/j.1360-0443.2008.02514.x

Nam, E., \& Fukui, S. (2017). Tobacco, alcohol, and drug use of people with mood and anxiety disorders: differential impact of family and friends. Journal of dual diagnosis, 13(2), 124-132.

https://doi.org/10.1080/15504263.2017.1285092

Nurmi, J. E. (2004). Socialization and self-development channeling, selection, adjustment and reflection. Richard M. Lerner and Laurence Steinberg (eds.) Handbook of adolescent psychologhy (second Edition) (85-124). New Jersey: John Wiley\&Sons, Inc. https://doi.org/10.1002/9780471726746.ch4

Paget, A., Parker, C., Heron, J., Logan, S., Henley, W., Emond, A., \& Ford, T. (2018). Which children and young people are excluded from school? Findings from a large British birth cohort study, the A von L ongitudinal Study of Parents and C hildren (ALSPAC). Child: care, health and development, 44(2), 285-296. https://doi.org/10.1111/cch.12525

Pajares, F. (1996). Self-efficacy beliefs in academic settings. Review of Educational Research, 66(4), 543-578. https://doi.org/10.3102/00346543066004543

Pajares, F., \& Schunk, D. (2001). The development of academic self-efficacy. Development of Achievement Motivation. United States, 7. https://doi.org/10.1016/B978-012750053-9/50003-6

Peh, C. X., Shahwan, S., Fauziana, R., Mahesh, M. V., Sambasivam, R., Zhang, Y., ... Subramaniam, M. (2017). Emotion dysregulation as a mechanism linking child maltreatment exposure and self-harm behaviors in adolescents. Child Abuse \& Neglect, 67, 383-390. https://doi.org/10.1016/j.chiabu.2017.03.013

Peplak, J., Song, J. H., Colasante, T., \& Malti, T. (2017). “Only you can play with me!” Children's inclusive decision making, reasoning, and emotions based on peers' gender and behavior problems. Journal of Experimental Child Psychology, 162, 134-148. https://doi.org/10.1016/j.jecp.2017.04.019

Plenty, S., \& Mood, C. (2016). J. Youth Adolescence, 45, 1294. https://doi.org/10.1007/s10964-016-0430-5

Prinstein, M. J., Heilbron, N., Guerry, J. D., Franklin, J. C., Rancourt, D., Simon, V., \& Spirito, A. (2010). Peer Influence and Nonsuicidal Self Injury: Longitudinal Results in Community and Clinically-Referred Adolescent Samples, J. Abnorm. Child Psychol. 38, 669. https://doi.org/10.1007/s10802-010-9423-0

Richter, M. (2010). Risk behaviour in adolescence. Springer Fachmedien. https://doi.org/10.1007/978-3-531-92364-2

Rose, A. J., \& Rudolph, K. D. (2006). A review of sex differences in peer relationship processes: potential trade-offs for the emotional and behavioral development of girls and boys. Psychological Bulletin, 132(1), 98. https://doi.org/10.1037/0033-2909.132.1.98

Rusby, J. C., Forrester, K. K., Biglan, A., \& Metzler, C. W. (2005). Relationships between peer harassment and adolescent problem behaviors. The Journal of Early Adolescence, 25(4), 453-477. https://doi.org/10.1177/0272431605279837

Sarıçam, H., Akın, A., Akın, Ü., \& Çardak, M. (2013). Algılanan Sosyal Yetkinlik Ölçeğinin Türkçeye uyarlanması: Geçerlik ve güvenirlik çalışması. The Journal of Academic Social Science Studies, 6(3), 591-600.

Schunk, D. H., \& Judith, L. M. (2005). Self-efficacy development in adolesence. Tim Urdan and Frank Pajares (Eds.) Self-Efficacy beliefs of adolescence (71-96). USA: Information Age Publishing.

Schwartz-Mette, R. A., \& Smith, R. L. (2018). When does co-rumination facilitate depression contagion in adolescent friendships? Investigating intrapersonal and interpersonal factors. Journal of Clinical Child \& Adolescent Psychology, 47(6), 912-924. https://doi.org/10.1080/15374416.2016.1197837

Segrin, C., \& Flora, J. (2011). Family Communication. Routledge. https://doi.org/10.4324/9780203857830

Shope, J. T., Waller, P. F., Raghunathan, T. E., \& Patil, S. M. (2001). Adolescent antecedents of high-risk driving behavior into young adulthood: substance use and parental influences. Accident Analysis \& Prevention, 33(5), 649-658. https://doi.org/10.1016/S0001-4575(00)00079-8

Silvers, J. A., McRae, K., Gabrieli, J. D., Gross, J. J., Remy, K. A., \& Ochsner, K. N. (2012). Age-related differences in emotional reactivity, regulation, and rejection sensitivity in adolescence. Emotion, 12(6), 1235. https://doi.org/10.1037/a0028297

Siyez, D. M. (2008). Adolescent self-esteem, problem behaviors, and perceived social support in Turkey. Social 
Behavior and personality: an international journal, 36(7), 973-984. https://doi.org/10.2224/sbp.2008.36.7.973

Siyez, D. M., \& Aysan, F. (2007). Ergenlerde görülen problem davranışların psiko-sosyal risk faktörleri ve koruyucu faktörler açısından yordanması. Uludağ Üniversitesi Eğitim Fakültesi Dergisi, 20(1), 145-171.

Susman, E., \& Rogal, A. (2004). Puberty and Psychological Development. Richard M. Lerner and Laurence Steinberg (Eds.) Handbook of adolescent psychology (second Edition) (15-45). New Jersey: John Wiley\&Sons, Inc. https://doi.org/10.1002/9780471726746.ch2

Taliaferro, L. A., Muehlenkamp, J. J., Borowsky, I. W., McMorris, B. J., \& Kugler, K. C. (2012). Factors distinguishing youth who report self-injurious behavior: A population-based sample. Academic pediatrics, 12(3), 205-213. https://doi.org/10.1016/j.acap.2012.01.008

Thompson, R. A. (1991). Emotional regulation and emotional development. Educational Psychology Review, 3(4), 269-307. https://doi.org/10.1007/BF01319934

Tomé, G., de Matos, M. G., Simões, C., Camacho, I., \& AlvesDiniz, J. (2012). How can peer group influence the behavior of adolescents: explanatory model. Global journal of health science, 4(2), 26. https://doi.org/10.5539/gjhs.v4n2p26

Vanderbilt, D., \& Augustyn, M. (2010). The effects of bullying. Paediatrics and child health, 20(7), 315-320. https://doi.org/10.1016/j.paed.2010.03.008

Veenstra, R., Dijkstra, J. K., \& Kreager, D. A. (2018). Pathways, networks, and norms: A sociological perspective on peer research. In W. M. Bukowski, B. Laursen, \& K. H. Rubin (Eds.), Handbook of peer interactions, relationships, and groups (pp. 45-63). New York, NY, US: The Guilford Press.

Wang, M., Liu, S., Zhan, Y., \& Shi, J. (2010). Daily work-family conflict and alcohol use: Testing the cross-level moderation effects of peer drinking norms and social support. Journal of Applied Psychology, 95(2), 377. https://doi.org/10.1037/a0018138

Wilkinson, R. G., \& Marmot, M. (Eds.). (2003). Social determinants of health: the solid facts. World Health Organization.

Wilson, R. J., \& Jonah, B. A. (1988). The application of Problem Behavior Theory to the understanding of risky driving. Alcohol, Drugs \& Driving, 4(3-4), 173-191.

Windle, M., Spear, L. P., Fuligni, A. J., Angold, A., Brown, J. D., Pine, D., ... Dahl, R. E. (2008). Transitions into underage and problem drinking: developmental processes and mechanisms between 10 and 15 years of age. Pediatrics, 121(Supplement 4), 273-289. https://doi.org/10.1542/peds.2007-2243C

Yeager, D. S. (2017). Social and emotional learning programs for adolescents. The Future of Children, 73-94. https://doi.org/10.1353/foc.2017.0004

Yurgelun, T. D. (2007). Emotional and cognitive changes during adolescence. Current Opinion in Neurobiology, 17(2), 251-257. https://doi.org/10.1016/j.conb.2007.03.009

Zimmerman, B. J., \& Timothy, J. C. (2005). Adolescent's development of personal agency, The role of self-efficacy beliefs and self regulatory skill. Tim Urdan and Frank Pajares (Ed.) Self efficacy beliefs of adolescence, (45-69). USA: Information Age Publishing.

\section{Copyrights}

Copyright for this article is retained by the author(s), with first publication rights granted to the journal.

This is an open-access article distributed under the terms and conditions of the Creative Commons Attribution license which permits unrestricted use, distribution, and reproduction in any medium, provided the original work is properly cited. 